\title{
Tonabersat Prevents Inflammatory Damage in the Central Nervous System by Blocking Connexin 43 Hemichannels
}

\author{
Yeri Kim ${ }^{1,2}$ • Jarred M. Griffin ${ }^{3}$ - Mohd N. Mat Nor ${ }^{2,4,7}$ • Jie Zhang ${ }^{1,2}$. \\ Peter S. Freestone $^{5}$ • Helen V. Danesh-Meyer ${ }^{1,2}$ • Ilva D. Rupenthal ${ }^{1,2,6}$. \\ Monica Acosta ${ }^{2,4}$ - Louise F. B. Nicholson ${ }^{3}$ - Simon J. O'Carroll ${ }^{3}$ - Colin R. Green ${ }^{1,2}$
}

Published online: 30 May 2017

(C) The American Society for Experimental NeuroTherapeutics, Inc. 2017

\begin{abstract}
The cis benzopyran compound tonabersat (SB220453) has previously been reported to inhibit connexin 26 expression in the brain by attenuating the $\mathrm{p} 38$-mitogenactivated protein kinase pathway. We show here that tonabersat directly inhibits connexin 43 hemichannel opening. Connexin 43 hemichannels have been called "pathological pores" based upon their role in secondary lesion spread, edema, inflammation, and neuronal loss following central nervous system injuries, as well as in chronic inflammatory disease. Both connexin 43 hemichannels and pannexin channels released adenosine triphosphate (ATP) during ischemia in an in vitro ischemia model, but only connexin 43 hemichannels contributed to ATP release during reperfusion. Tonabersat inhibited connexin 43 hemichannel-
\end{abstract}

Electronic supplementary material The online version of this article (doi:10.1007/s13311-017-0536-9) contains supplementary material, which is available to authorized users.

Colin R. Green

c.green@auckland.ac.nz

1 Department of Ophthalmology, Faculty of Medical and Health Sciences, University of Auckland, Auckland 1142, New Zealand

2 New Zealand National Eye Centre, Faculty of Medical and Health Sciences, University of Auckland, Auckland 1142, New Zealand

3 Centre for Brain Research, Faculty of Medical and Health Sciences, University of Auckland, Auckland 1142, New Zealand

4 School of Optometry and Vision Science, Faculty of Medical and Health Sciences, University of Auckland, Auckland 1142, New Zealand

5 Department of Physiology, Faculty of Medical and Health Sciences, University of Auckland, Auckland 1142, New Zealand

6 Buchanan Ocular Therapeutics Unit, Faculty of Medical and Health Sciences, University of Auckland, Auckland 1142, New Zealand

7 Faculty of Medicine, University Sultan Zainal Abidin, Kuala Terengganu, Malaysia mediated ATP release during both ischemia and reperfusion phases, with direct channel block confirmed using electrophysiology. Tonabersat also reduced connexin 43 gap junction coupling in vitro, but only at higher concentrations, with junctional plaques internalized and degraded via the lysosomal pathway. Systemic delivery of tonabersat in a rat bright-light retinal damage model (a model for dry age-related macular degeneration) resulted in significantly improved functional outcomes assessed using electroretinography. Tonabersat also prevented thinning of the retina, especially the outer nuclear layer and choroid, assessed using optical coherence tomography. We conclude that tonabersat, already given orally to over 1000 humans in clinical trials (as a potential treatment for, and prophylactic treatment of, migraine because it was thought to inhibit cortical spreading depression), is a connexin hemichannel inhibitor and may have the potential to be a novel treatment of central nervous system injury and chronic neuroinflammatory disease.

Keywords Tonabersat $\cdot$ connexin $43 \cdot$ connexin hemichannel $\cdot$ gap junction $\cdot$ CNS disease

\section{Introduction}

Tonabersat (SB-220453), a novel cis benzopyran derivative, was selected for its efficacy against neuronal hyperexcitability and neurogenic inflammation in a structure-function study of antimigraine compounds [1]. Tonabersat was said to prevent the activation of nitric oxide and elevation of cyclic guanosine monophosphate associated with neuronal depolarization and sensitization of trigeminal nerves in vivo by binding to a stereoselective site in the central nervous system (CNS) [2]. Tonabersat was also reported to inhibit cortical spreading depression (CSD), a key mechanism underlying the depolarizing brain waves in a migraine with aura [3]. Features of CSD are 
also reported in secondary tissue damage following stroke [4], epilepsy [5], and brain injury [6]. Based on preclinical results, tonabersat was selected for phase II clinical trials as a prophylactic treatment for migraines [7, 8], where it was well tolerated and devoid of adverse effects in the cardiovascular system [9]. It was also proposed as a treatment for epilepsy [10]. While the clinical results for tonabersat were still under evaluation [11], the cell-to-cell signaling involved in CSD gave rise to gap junctions (GJs), which are intercellular channels, as possible therapeutic targets for tonabersat for CSD [12]. GJ channels are from a highly conserved family of connexin proteins, and connexin43 is a major subtype of the CNS [13-16]. Structurally, connexin proteins are composed of 4 transmembrane domains, cytoplasmic $N$ - and $C$-termini, a cytoplasmic loop, and 2 highly conserved extracellular loops [17]. The oligomerization of 6 connexin proteins forms a connexon [18], or "hemichannel", that aligns and docks with an adjacent hemichannel in a neighboring cell to form an intercellular GJ channel [19]. In physiology and pathology, GJ channels coordinate cellular behavior by providing a direct and lowresistance intercellular pathway for molecules up to $1 \mathrm{kDa}$ $[20,21]$. An open connexin 43 hemichannel, however, constitutes a large and relatively nonspecific pore between the intracellular space and extracellular milieu. Specific physiological roles for connexin 43 hemichannels have been proposed [22-24], but these channels also have a low basal opening probability [25]. Additionally, conditioning factors such as changes in $\mathrm{Ca}^{2+}$ concentration [increase in cytoplasmic $\mathrm{Ca}^{2+}$ concentration or decrease in extracellular $\mathrm{Ca}^{2+}$ concentration $\left.\left(\mathrm{Ca}^{2+}{ }_{\mathrm{o}}\right)\right][19,26-28]$, metabolic inhibition [29, 30], reduced redox potential [31], and membrane depolarization [25, 32] have all been shown to increase the opening probability of connexin43 hemichannels. Enhanced connexin 43 hemichannel activity has been reported to accelerate cell death by compromising metabolic, ionic, and energy gradients [33]. Open hemichannels have also been suggested to contribute to cell swelling [34], excitotoxic cell death [35], and vascular hemorrhage [36, 37]. Furthermore, adenosine triphosphate (ATP) released from unregulated connexin 43 hemichannels has been suggested to activate the purinergic cascade [38], which is reported to be a signaling pathway associated with the inflammasome pathway leading to the maturation and secretion of proinflammatory cytokines such as interleukin (IL)$1 \beta$ and IL-18 from inflammatory cells $[39,40]$.

Hemichannels also propagate secondary damage signals following spinal cord injury [41-44], CNS ischemia [35, 37, 45-48], and retinal injuries [49, 50], and have been termed "pathological pores" $[16,51]$. Upregulation of the expression of connexin43 following an insult is also reported to exacerbate the extent of secondary damage [37, 43, 46, 52-55].

Modulating connexin 43 hemichannel opening under cellular stress has been shown to avert secondary injury mechanisms. In the retina, blocking connexin 43 hemichannel activity with a low concentration of connexin mimetic peptide (Peptide5) [40, 56], a mimicking sequence of the second extracellular loop of connexin43, significantly reduced vascular leak, vessel loss, and retinal ganglion cell death in a model of retinal ischemiareperfusion [37]. Peptide5 also reduced inflammation, improved functional outcomes, and retained retinal tissue integrity following bright-light damage used as a model for dry age-related macular degeneration [50]. In other areas of the CNS, Peptide5 improved functional outcomes following spinal cord injury [ 43 , 44], and in sheep models of perinatal ischemia and asphyxia by reducing seizures and significantly improving electroencephalographic power $[46,47]$. Other connexin43 extracellular loopmimicking peptides such as Gap26 [57] and Gap27 [52], and an intracellular acting mimetic peptide, Gap19 [58], have also been reported to limit cell death by reducing connexin 43 hemichannel activity following injury. In a different approach, connexin 43 antisense oligodeoxynucleotides (a single strand of DNA) promoted wound healing by specifically inhibiting connexin43 synthesis [59-61]. The protective effect of connexin 43 antisense oligodeoxynucleotides likely involves transient attenuation in the expression of hemichannels and GJ channels, although hemichannels may be the primary contributors to secondary injury effects.

Tonabersat has previously been reported to inhibit connexin26 GJ formation between trigeminal neurons and satellite glial cells by attenuating the p38-mitogen-activated protein kinase (MAPK) pathway [12], a signal transduction cascade involved in inflammation and apoptosis [62]. To the best of our knowledge though there has been no report showing that tonabersat is connexin26-specific. Tonabersat is known to bind with high affinity to a stereoselective yet unspecified site in the CNS [1]. Connexin43 is abundantly expressed in the CNS [13-15], and we investigated whether tonabersat could improve functional outcomes in in vitro and in vivo models of CNS injury and disease by modulating connexin 43 channels.

Using an in vitro ischemia-reperfusion model we characterized the concentration- and time-dependent properties of tonabersat and sought to determine if it can specifically target connexin43 hemichannels without affecting GJ communication. We then translated our in vitro results into an established in vivo model of retinal injury - the bright-light damage model $[37,50,54]$. This in vivo model enabled us to assess whether tonabersat can protect against functional loss and make direct comparisons with previously published connexin 43 mimetic peptide (Peptide5) intervention studies [50]. Here we show that a low concentration of tonabersat can attenuate ATP release from connexin 43 hemichannels following injury and reperfusion conditions in vitro, indicating direct hemichannel block. Electrophysiology showed that channel closure occurs directly $(<3 \mathrm{~min})$. High concentrations of tonabersat, however, reduced GJ coupling in a scrape-load dye-spread model (down to about half), with sustained long-term exposure 
causing internalization and degradation of membrane connexin43 plaques via the lysosomal pathway. This study demonstrates that tonabersat is not only acting on connexin26 GJ formation or glia neurons as previously reported, but that it can also inhibit connexin 43 hemichannels in other cell types and can partially regulate connexin 43 GJ coupling at higher concentrations. Our results support the investigation of tonabersat as a potential treatment to prevent hemichannelmediated inflammatory damage and/or vessel loss, both of which are common to acute injuries of the CNS such as trauma, stroke, and ischemia, and to block perpetuation of the inflammasome pathway in chronic neuroinflammatory diseases such as age-related macular degeneration, diabetic retinopathy, chronic pain, Alzheimer's disease, and Parkinson's disease.

\section{Methods}

\section{Cell Preparation for in vitro Assays}

Human cerebral microvascular endothelial cells (hCMVEC) (ABM, Milton, ON, Canada), which have been characterized extensively for their endothelial phenotype in terms of their cytokine secretion and cell-surface adhesion molecules [63], were grown in collagen-coated $\left(30 \mu \mathrm{g} / \mathrm{cm}^{2}\right) \mathrm{T} 25$ or T75 flasks and maintained in growing medium M199 supplemented with $10 \%$ fetal calf serum, $1 \mu \mathrm{g} / \mathrm{ml}$ hydrocortisone, $3 \mathrm{ng} / \mathrm{ml}$ human fibroblast growth factor, $1 \mathrm{ng} / \mathrm{ml}$ human epidermal growth factor, $10 \mu \mathrm{g} / \mathrm{ml}$ heparin, $1 X$ penicillin streptomycin neomycin (Life Technologies, Carlsbad, CA, USA), and $8 \mathrm{nM}$ cyclic adenosine monophosphate. Cells were maintained at $37^{\circ} \mathrm{C}$ with $95 \% \mathrm{O}_{2}$ and $5 \% \mathrm{CO}_{2}$. Human retinal pigment epithelial (ARPE-19) cells were grown in DMEM/F:12 (Invitrogen, Carlsbad, CA, USA), supplemented with $10 \%$ fetal calf serum, $100 \mathrm{units} / \mathrm{ml}$ of penicillin, $100 \mu \mathrm{g} / \mathrm{ml}$ streptomycin, and $0.25 \mu \mathrm{g} / \mathrm{ml}$ Fungizone ${ }^{\circledR}$ Antimycotic (Invitrogen).

\section{Chemicals}

Tonabersat (Medchemexpress, Monmouth Junction, NJ, USA) was dissolved in dimethyl sulfoxide (DMSO) at a stock concentration of $100 \mathrm{mM}$ for in vitro studies. Ammonium chloride $\left(\mathrm{NH}_{4} \mathrm{Cl}\right.$; Sigma, St. Louis, MO, USA) was dissolved in ultrapure water $\left(\mathrm{H}_{2} \mathrm{O}\right)$ at a stock concentration of $100 \mathrm{mM}$. Connexin43 channel-inhibiting mimetic peptide (Peptide5, sequence VDCFLSRPTEKT) (Auspep, Tullamarine, VIC, Australia), was synthesized at a purity of $>95 \%$ and dissolved in ultrapure $\mathrm{H}_{2} \mathrm{O}$ at a concentration of $10 \mathrm{mM}$. Probenecid $\left(\mathrm{C}_{13} \mathrm{H}_{19} \mathrm{NO}_{4} \mathrm{~S}\right.$; Sigma), solubilized in $1 \mathrm{M} \mathrm{NaOH}$ at a concentration of $50 \mathrm{mg} / \mathrm{ml}$, was used to block Pannexin channel opening in order to characterize connexin43 hemichannels in isolation. Carbenoxolone (CBX; Sigma), a nonspecific inhibitor of connexin and pannexin channels, and a broadspectrum inhibitor of GJ channels [64], was dissolved in ultrapure $\mathrm{H}_{2} \mathrm{O}$ at a stock concentration of $10 \mathrm{mM}$. For in vivo studies, tonabersat was dissolved in $60 \%$ cyclodextrin and $40 \%$ polyethylene glycol (PEG). Briefly, $2.4 \mathrm{ml} \mathrm{Milli-Q^{ \circledR }}{ }^{\text {wa- }}$ ter (Millipore, Billerica, MA, USA) was added to $3.6 \mathrm{~g}$ cyclodextrin and placed on a rocker for several hours until dissolved. Then, Milli-Q water was added up to $6 \mathrm{ml}$ followed by $4 \mathrm{ml}$ PEG and mixed thoroughly. A mass of $10 \mathrm{mg}$ tonabersat was added to $10 \mathrm{ml}$ of (2-hyrdoxypropyl)- $\beta$-cyclodextrin (Sigma Aldrich, St. Louis, MO, USA) and PEG (MW400) (Sigma Aldrich) vehicle mixture and sonicated at $35-40^{\circ} \mathrm{C}$ in a sonicating water bath for $2 \mathrm{~h}$ until the Tonabersat was well mixed with the vehicle.

\section{In vitro Injury-Reperfusion ATP Assay}

hCMVEC were trypsinized (TrypLE Express; Life Technologies) and plated at a density of $0.025 \times 10^{6}$ cells per well in a collagen-coated 12-well plate $\left(1 \mu \mathrm{g} / \mathrm{cm}^{2}\right)$ a day prior to the experiment. Cells were incubated in culture medium overnight. Hypoxic, acidic, ion-shifted Ringer injury (HAIR) solution that mimics ionic concentrations and acidbase shifts of the interstitial space in hypoxic-ischemic brains [65] was used to trigger hemichannel opening. The injury solution contained $38 \mathrm{mM} \mathrm{NaCl}, 13 \mathrm{mM} \mathrm{NaHCO}, 3 \mathrm{mM}$ Na-gluconate, $65 \mathrm{mM} \mathrm{K}$-gluconate, $38 \mathrm{mM}$ NMDG-Cl, $1 \mathrm{mM} \mathrm{NaH}_{2} \mathrm{PO}_{4}$, and $1.5 \mathrm{mM} \mathrm{MgCl}_{2}$. The injury solution was bubbled in $\mathrm{N}_{2}$ gas (> 99\% purity, $201 / \mathrm{min}$ ) for $5 \mathrm{~min}$ and $\mathrm{pH}$ adjusted to 6.6 with $5 \mathrm{M} \mathrm{HCl}$ before use. The standard Ringer solution contained $124 \mathrm{mM} \mathrm{NaCl}, 3 \mathrm{mM} \mathrm{KCl}, 26 \mathrm{mM}$ $\mathrm{NaHCO}_{3}, 1 \mathrm{mM} \mathrm{NaH} \mathrm{PO}_{4}, 1.3 \mathrm{mM} \mathrm{CaCl} 2,1.5 \mathrm{mM} \mathrm{MgCl}_{2}$, and $10 \mathrm{mM}$ glucose, and $\mathrm{pH}$ adjusted to 7.4 with $5 \mathrm{M} \mathrm{HCl}$ before use [65]. In the ischemia injury model, hCMVEC were incubated for $2 \mathrm{~h}$ in tonabersat $(0.1-100 \mu \mathrm{M}) \pm$ probenecid $(1$ $\mathrm{mM})$ dissolved in injury solution $(500 \mu \mathrm{l})$. In the ischemiareperfusion model, hCMVEC were first incubated in injury solution for $2 \mathrm{~h}$, which was discarded and replaced with tonabersat $(10 \mu \mathrm{M})$ dissolved in standard Ringer solution $(500 \mu \mathrm{l})$ for a further $2 \mathrm{~h}$. In ischemia or ischemia-reperfusion models, hCMVEC were incubated in standard Ringer solution only as a negative control. Injury solution or injury solution followed by standard Ringer solution was used as a positive control for ischemia injury and ischemia-reperfusion models, respectively. Established inhibitors, Peptide5 $(100 \mu \mathrm{M})$ [37, $40,56]$ and probenecid $(1 \mathrm{mM})[66]$, were used to differentiate connexin 43 and pannexin activity for both injury and injuryreperfusion models. CBX at a concentration of $100 \mu \mathrm{M}$ was also used. These established inhibitors enabled us to compare whether tonabersat was targeting connexin hemichannels and/ or pannexin channels. All incubations were conducted at $37^{\circ} \mathrm{C}$ in $95 \% \mathrm{O}_{2}$ and $5 \% \mathrm{CO}_{2}$. At the end of each experiment incubating solutions were removed and immediately placed on ice. 
The concentration of ATP in the solutions was determined using a luciferin/luciferase bioluminescence reaction (ATP Determination Kit; Molecular Probes, Eugene, OR, USA) and detected using a luminescence plate reader (VICTOR X; Perkin Elmer \#2030-0010; Waltham, MA, USA). Standard curves were generated in each experiment from an ATP standard $(0-500 \mathrm{nM})$ to quantify the concentration of ATP in the test samples. Treatment groups had a sample size of 2 wells per experiment, and the concentration of ATP in each sample was measured in triplicates over 10 repeated readings. The data are presented as mean $\pm \mathrm{SE}$ relative to the injury or injury-reperfusion-positive control. Statistically significant differences between samples were tested using 1-way analysis of variance (ANOVA) and Tukey's multiple comparisons test.

To ensure that none of the drugs or vehicles used could directly affect the enzymatic ATP assay, ATP standard curves were also run (3 repeats of each) in HAIR, HAIR + tonabersat $(100 \mu \mathrm{M})$, normal Ringer, HAIR + probenecid $(1 \mathrm{mM})$, HAIR $+\mathrm{CBX}(100 \mu \mathrm{M})$, HAIR + peptide $5(100 \mu \mathrm{M})$, and HAIR + vehicle (DMSO).

\section{Electrophysiology}

hCMVEC were plated at $0.045 \times 10^{6}$ cells $/ \mathrm{ml}$ on sterile 18 $\mathrm{mm}$-diameter rat collagen I coated coverslips 1 day before the experiment in growing medium at $37^{\circ} \mathrm{C}$ at $95 \% \mathrm{O}_{2}$ and $5 \%$ $\mathrm{CO}_{2}$. The next day cells were perfused with artificial cerebrospinal fluid (aCSF) containing $125 \mathrm{mM} \mathrm{NaCl}, 25 \mathrm{mM}$ $\mathrm{NaHCO}_{3}, 3 \mathrm{mM} \mathrm{KCl}, 1.25 \mathrm{mM} \mathrm{NaH} \mathrm{PO}_{4}, 1 \mathrm{mM} \mathrm{MgCl}$, $1.8 \mathrm{mM} \mathrm{CaCl}_{2}$, and $10 \mathrm{mM}$ glucose bubbled in $95 \% \mathrm{O}_{2}$ and $5 \% \mathrm{CO}_{2}$ at room temperature. The internal pipette solution contained $130 \mathrm{mM} \mathrm{KCl}, 10 \mathrm{mM}$ sodium aspartate, $0.26 \mathrm{mM}$ $\mathrm{CaCl}_{2}, 2 \mathrm{mM}$ EGTA, $5 \mathrm{mM}$ tetraethylammonium-Cl, $1 \mathrm{mM}$ $\mathrm{MgCl}, 3 \mathrm{mM} \mathrm{MgATP}$, and $5 \mathrm{mM}$ HEPES at $\mathrm{pH} 7.2$ [25], and kept on ice. High-resistance pipettes of 6 to $10 \mathrm{M} \Omega$ were pulled from Corning 7056 patch glass capillaries (outer diameter $1.5 \mathrm{~mm}$, inner diameter $1.1 \mathrm{~mm}$, wall thickness $0.4 \mathrm{~mm}$ ) using a P-97 electrode puller (Sutter Instruments, Novato, CA, USA). To resolve single currents in the whole-cell configuration [67], isolated cells with low capacitance $(<10 \mathrm{pF})$ with a seal resistance of $>5 \mathrm{G} \Omega$ were selected for recording. A voltage clamp was used to elicit hemichannel currents by holding the membrane potential $(\mathrm{Vm})$ at $0 \mathrm{mV}$ for $100 \mathrm{~ms}$, followed by positive voltage steps of $10 \mathrm{mV}$ to $80 \mathrm{mV}$ in $10 \mathrm{mV}$ increments at $15 \mathrm{~s}$ each. Voltage-clamp recording and drug perfusion were conducted simultaneously. Cells were perfused with aCSF for $1 \mathrm{~min}$; then, channel currents were recorded for 1 min following 5 min perfusion with Tonabersat $(50 \mu \mathrm{M})$ in aCSF. Tonabersat was washed out for 5 min before a 1min recording of the washout period. Currents were measured using amplifier (MultiClamp 700B; Molecular Devices, Sunnyvale, CA, USA) and digitizer (Digidata, 1200B; Axon
Instruments from Molecular Devices). The data were low-pass filtered at $1 \mathrm{kHz}$ and digitized at sampling intervals of $0.25 \mathrm{~ms}$ using pClamp 10 software (Molecular Devices).

\section{Scrape Loading GJ Assay}

hCMVEC were trypsinized (TrypLE Express; Life Technologies) and plated at a density of $0.4 \times 10^{6}$ cells per well in a collagen-coated 12-well plate, and incubated in culture medium overnight. Once confluency was reached, the cells were preincubated in tonabersat $(0.1,1,10,50$, or 200 $\mu \mathrm{M})$ dissolved in culture medium for $1 \mathrm{~min}$, or 1, 2, 6, or $24 \mathrm{~h}$. $\mathrm{CBX}$ at a concentration of $200 \mu \mathrm{M}$ was used as a positive control. Cells were washed 3 times with phosphate-buffered saline (PBS) without $\mathrm{Ca}^{2+}$ or $\mathrm{Mg}^{2+}$. The cells were then incubated in $0.05 \%$ Lucifer Yellow (LY) (Sigma) \pm tonabersat dissolved in PBS, and scrape-wounded with a size 10 carbon-steel surgical blade. Following 5 min incubation at $37^{\circ} \mathrm{C}$ in $95 \% \mathrm{O}_{2}$ and $5 \% \mathrm{CO}_{2}$ without light, the $0.05 \% \mathrm{LY}$ solution was removed. Cells were rinsed 4 times with PBS with $\mathrm{Ca}^{2+}$ or $\mathrm{Mg}^{2+}$, and then fixed in $4 \%$ paraformaldehyde (PFA) (ProSciTech, Kirwan, QLD, Australia) in PBS at $\mathrm{pH}$ 7.4 for $10 \mathrm{~min}$ at room temperature. Cells were then washed 3 times in PBS to remove PFA before fluorescent imaging. LY was visualized using a Nikon TE2000E inverted fluorescent microscope (10× magnification, 0.3 numerical aperture), and captured using a Digital Sight CCD camera and Eclipse Net software (Nikon, Tokyo, Japan). Three images within each well from 3 independent experiments were taken for analysis. The Transfluor feature in MetaXpress ${ }^{\circledR}$ Image acquisition and analysis software (version 5.3.0.1; Molecular Devices) was used to quantify the total number of LY-positive cells. The data are presented as means \pm SE relative to the no-treatment control. Statistically significant differences between samples in a given experiment were tested using 1-way ANOVA with the Tukey's multiple comparisons test $(n=3$ independent experiments, 3 wells).

\section{Immunocytochemistry and Quantification of Connexin43 Plaques}

ARPE-19 cells were grown until confluent in 8-well glass chamber-slides (BD Falcon). Confluent monolayers of ARPE-19 cells were incubated at a final concentration of 5, $10,50,100,200$, and $500 \mu \mathrm{M}$ tonabersat, and/or $10 \mathrm{mM}$ $\mathrm{NH}_{4} \mathrm{Cl}$ [68] in culture medium for 1 or $6 \mathrm{~h}$. Cells were fixed in $4 \%$ PFA at $\mathrm{pH} 7.4$ for $10 \mathrm{~min}$ at room temperature, permeabilized with $0.05 \%$ Triton-X100 in PBS, and incubated in $10 \%$ normal goat serum to block nonspecific labeling. Cells were rinsed 3 times with PBS containing $0.1 \mathrm{mM} \mathrm{CaCl}_{2}$ between each fixation, permeabilization, and blocking steps. Connexin43 polyclonal rabbit antibody (C6219, 1:2000; Sigma) was applied for $24 \mathrm{~h}$, followed by a goat antirabbit 
Alexa Fluor ${ }^{\circledR} 568$ secondary antibody (1:200; Invitrogen) for $45 \mathrm{~min}$. Nuclei were counterstained with 4',6-diamidino-2phenylindole (Invitrogen) at 10,000-fold dilution for $5 \mathrm{~min}$, and mounted with Citifluor ${ }^{\mathrm{TM}}$ mounting medium. Images were visualized and captured using an oil-immersion lens (60× magnification, 1.35 numerical aperture) on an Olympus FV1000 upright confocal laser scanning microscope and Olympus FV10-ASW 4.0 software. The Transfluor feature in MetaXpress ${ }^{\circledR}$ Image acquisition and analysis software was used to quantify the total area of connexin 43 plaques per image. Results represent mean $\pm \mathrm{SE}$ and statistical tests were conducted using 1-way ANOVA and Tukey's multiple comparisons test $(n=3$ wells in each treatment from 2 independent experiments).

\section{Real-Time Reverse Transcription Polymerase Chain Reaction}

Real-time reverse transcription polymerase chain reaction (PCR) was used to determine the relative levels of connexin43 mRNA. A confluent monolayer of ARPE-19 cells was treated with $50 \mu \mathrm{M}$ Tonabersat or $0.05 \%$ DMSO vehicle control for 1 h. Cells without treatment were also used as a further control. Cells were harvested with TRIzol ${ }^{\circledR}$ (Life Technologies), and total RNA was isolated using SuperScript III First Strand kit (Invitrogen) according to the manufacturer's instructions. All RNA samples were quantified using a nanodrop 1000 microvolume spectrophotometer (Thermo Scientific, Waltham, MA, USA). cDNAs from samples were synthesized using SuperScript ${ }^{\circledR}$ III First-Strand Synthesis SuperMix (Invitrogen) according to the manufacturer's instructions. Primer sequences for human connexin43, GenBank Accession No. M65188.1, were obtained from the Harvard Primer bank. The sequences for human connexin 43 were forward 5'-TGGTAAGGTGAAAATGCGAGG-3' and reverse 5'-GCACTCAAGCTGAATCCATAGAT-3'. The appropriate reference gene to be used for relative fold-difference in connexin 43 mRNA was determined by screening commonly used housekeeping genes, $\beta$-actin (ACTB), hypoxanthine phosphoribosyltransferase 1 (HPRT1), peptidylprolyl isomerase A (PPIA), and glyceraldehyde-3-phosphate dehydrogenase $(G A P D H)$. We chose $\beta$-actin as the control gene as it showed no significant difference in expression between the treatment groups. All PCR reactions were performed on the Rotor gene600 (Qiagen, Hilden, Germany) using a cycle program of $50^{\circ} \mathrm{C}$ for $2 \mathrm{~min}$ then $95^{\circ} \mathrm{C}$ for $2 \mathrm{~min} ; 95^{\circ} \mathrm{C}$ for $15 \mathrm{~s}$ and $60^{\circ} \mathrm{C}$ repeated 40 times; $95^{\circ} \mathrm{C}$ for $15 \mathrm{~s}, 60^{\circ} \mathrm{C}$ for $15 \mathrm{~s}, 95^{\circ} \mathrm{C}$ for $15 \mathrm{~s}$, in order to generate threshold cycles for relative quantification $(\mathrm{Ct})$. Relative changes in connexin 43 mRNA expression were calculated as fold-differences against $\beta$-actin using the $2^{-\Delta \Delta \mathrm{Ct}}$ method. Results represent mean $\pm \mathrm{SE}$ and statistical tests were conducted using a 1-way ANOVA followed by Tukey's multiple comparisons test.

\section{3-(4,5-Dimethylthiazol-2-yl)-2,5-Diphenyltetrazolium Bromide Viability Assay}

hCMVEC were seeded in a collagen-coated 96 well-plate at 1 $\times 10^{4}$ cells/well and incubated in culture medium overnight. The following day, cells were washed twice with PBS and incubated in culture medium with tonabersat $(0.2,2,20$, and $200 \mu \mathrm{M}$ ) for $24 \mathrm{~h}$. Following incubation, the culture medium was removed and replaced with $100 \mu \mathrm{l}$ PBS. A volume of $10 \mu \mathrm{l}$ 3-(4,5-dimethylthiazol-2-yl)-2,5-diphenyltetrazolium bromide stock $(5 \mathrm{mg} / \mathrm{ml})$ was added to each well, except for the blank control, and was left to incubate at $37^{\circ} \mathrm{C}$ for $4 \mathrm{~h}$. The solution was then aspirated from each well and replaced with $50 \mu \mathrm{LMSO}$ (Sigma) as a solvent. Viability was determined by measuring the absorbance at $595 \mathrm{~nm}$ in a plate reader (OPTIMA FLUOstar; BMG), and each measurement was standardized to no-treatment control in each experiment. Results represent mean $\pm \mathrm{SE}$ and statistical tests were conducted using a 1-way ANOVA followed by Tukey's multiple comparisons test.

\section{Light-Damage Animal Model}

All procedures were conducted in compliance with the ARVO Statement of Use of Animals in Ophthalmic and Vision Research and were approved by the Animal Ethics Committee of the University of Auckland. Adult SpragueDawley (SD) rats (200-250 g, equal numbers of male and female) were obtained from the Vernon Janson Unit of the University of Auckland and housed in a $12 \mathrm{~h}$ light/dark cycle and received food and water ad libitum. To induce light damage rats were exposed to bright light for $24 \mathrm{~h}$. Light exposure started consistently around 9:00 a.m. to minimize damage caused by variations in time of the day exposure [69]. The light luminance was 2700 lux, created by placing fluorescent light lamps (Philips Master TLD 18W/965; Koninklijke Philips Electronics N.V., Amsterdam, the Netherlands) directly above the rat cages. The light source covered broadband fluorescence, from 380 to $760 \mathrm{~nm}$, with no extra heat generated. The average intensity at the top of the cage was 120 $\mathrm{W} / \mathrm{m}^{2}$. This broadband fluorescence light has been used in previous studies on albino rats $[54,70]$. Animals were able to move freely in the cage and had access to food and water at libitum. The light-damage process was continuous otherwise, except for a brief period during tonabersat injection. Lightdamaged SD rats with vehicle only injection were used as sham control animals. There were 6 animals per group with 12 eyes per group analyzed.

Tonabersat was administered as a double injection in the rat peritoneum, the first injection $2 \mathrm{~h}$ into the 24-h light-damage period and the second injection immediately after the 24-h light-damage procedure. The dose administered was $80 \mu \mathrm{l}$ of 
the $1 \mathrm{mg} / \mathrm{ml}$ solution, calculated to give a final circulating concentration of $10 \mu \mathrm{M}$ (assuming a 20-ml blood volume).

Electroretinogram recording (ERG) for assessment of retinal function was performed as described previously [54]. SD rats were dark-adapted overnight for 12 to $14 \mathrm{~h}$ before the ERG recording. The ERG baseline was recorded before light damage and after $24 \mathrm{~h}$, and 7 and 14 days of intense light damage. A dim red light generated by a light-emitting diode $(\lambda \max =650 \mathrm{~nm})$ was used during manipulations of darkadapted animals. The corneas were maintained hydrated with $1 \%$ carboxymethylcellulose sodium (Celluvis ${ }^{\circledR}$; Allergan, Parsippany-Troy Hills, NJ, USA) throughout the whole ERG recording. Right and left eye ERGs were recorded using animal gold-ring electrodes (Roland Consult, Brandenburg, Germany). The active electrode was U-shaped and was kept in contact with the eyelid-cornea interface. The inactive electrode was $\mathrm{V}$ shaped and hooked around the front teeth and was in contact with the wet tongue. Body temperature was kept approximately at $37^{\circ} \mathrm{C}$ to avoid temperature-driven ERG amplitude fluctuation. Full-field ERG responses were elicited by a twin-flash (0.8-ms stimulus interval) generated from a photographic flash unit (Nikon SB900 flash) via a Ganzfeld sphere. Recordings were performed in a Faraday cage to reduce electrical noise. The flash unit triggered paired flashes that had identical luminous energy. The rod and cone mixed response were recorded after the initial flash, and the response was subtracted from the second flash to represent function from the cone photoreceptors only. The results of ERG signals were amplified 1000 times by a Dual Bio Amp and waveforms were recorded using the Scope software (AD Instruments, Sydney, Australia). Published algorithms were used in the analysis of the amplitudes of a-wave and b-wave ERG for each eye [50, 54].

A spectral domain optical coherence tomography (Micron IV; Phoenix Research Labs, Pleasanton, CA, USA) approach was used to investigate the thickness of the retinal layers in tonabersat or sham-treated light-damaged rats. This procedure was executed immediately after ERG recordings under the same anesthesia and pupil dilation [54]. Rats were placed on a $37^{\circ} \mathrm{C}$ heating pad to maintain body temperature and to prevent the development of cold cataracts. Dilated eyes were covered with Poly Gel (containing $3 \mathrm{mg} / \mathrm{g}$ Carbomer; Alcon Laboratories, Fort Worth, TX, USA) to act as an immersion medium, bridging the optical coherence tomography (OCT) lens to the cornea for retinal imaging. StreamPix 6 software, version 7.2.4.2 (Phoenix Research Labs) was used for image acquisition. The spectral domain optical coherence tomography horizontal line B-scan had 2- $\mu \mathrm{m}$ axial resolutions and consisted of 1024 pixels per A-scan. Ten B-scans, acquired $2 \mathrm{~mm}$ from the optic nerve in the dorsal retina, were taken and averaged. Images were analyzed using InSight software, version 1.1.5207 (Phoenix Research Labs) to calculate the thickness of retinal and choroidal layers. The total thickness of the
Fig. 1 Tonabersat inhibits hemichannels and inhibits adenosine triphosphate (ATP) release from human cerebral microvascular endothelial cells (hCMVEC) in injury and reperfusion. (A) ATP released from hCMVEC following $2 \mathrm{~h}$ of exposure to injury and treatment with carbenoxolone (CBX), Peptide5, probenecid, and a combination of Peptide5 and probenecid in vitro. Quantification of total extracellular ATP release is presented as a percentage of the injury control for each treatment group. A significant reduction in ATP was present across all treatment groups compared with injury control: $100 \mu \mathrm{M}$ CBX, $100 \mu \mathrm{M}$ Peptide5 alone, $1 \mathrm{mM}$ probenecid alone, and $100 \mu \mathrm{M}$ Peptide5 in combination with $1 \mathrm{mM}$ probenecid; the latter group reducing total ATP release to the same level as CBX. (B) Quantification of total extracellular ATP release in hCMVEC presented as a percentage of the injury control for each treatment group. A significant reduction in ATP was present across all treatment groups: $100 \mu \mathrm{M} \mathrm{CBX}$ and $0.1 \mu \mathrm{M}$ to $100 \mu \mathrm{M}$ tonabersat in combination with $1 \mathrm{mM}$ probenecid. A significant difference was present between $10 \mu \mathrm{M}$ and $100 \mu \mathrm{M}$ tonabersat. (C) In the absence of probenecid there was no significant reduction in extracellular ATP in the presence of $0.1 \mu \mathrm{M}$ to $100 \mu \mathrm{M}$ tonabersat compared with injury control during ischemia. (D) ATP released from subconfluent hCMVEC following $2 \mathrm{~h}$ of exposure to injury then $2 \mathrm{~h}$ of exposure to reperfusion in vitro. ATP is quantified as a percentage of the injuryreperfusion (IR) control. A significant reduction in ATP was observed with $100 \mu \mathrm{M}$ CBX, $100 \mu \mathrm{M}$ Peptide5 alone, and $100 \mu \mathrm{M}$ Peptide5 combination with $1 \mathrm{mM}$ probenecid, but not with $1 \mathrm{mM}$ probenecid alone. A significant reduction in ATP was observed with $10 \mu \mathrm{M}$ tonabersat. (E) Tonabersat attenuates single-channel currents elicited by positive membrane potentials in hCMVEC. Cells were perfused with artificial cerebrospinal fluid (aCSF) during a 15-s voltage protocol to positive membrane potentials $(\geq 60 \mathrm{mV}$ ) to stimulate connexin43 hemichannel currents (aCSF, before tonabersat, upper panels). Tonabersat $(50 \mu \mathrm{M})$ dissolved in aCSF immediately attenuated singlechannel currents (aCSF $+50 \mu \mathrm{M}$ Tonabersat, blue panel). Single-channel currents were partially recoverable upon washout of tonabersat with aCSF (aCSF washout, bottom panel). C (black dotted lines) and $\mathrm{O}$ (red dotted lines) indicate closed and open state, respectively. The values next to the lines indicate starting current. Values represent mean $\pm \mathrm{SE}$. Oneway analysis of variance Tukey's multiple comparison test $* p<0.05$, $* * p$ $<0.01, * * * p<0.001, * * * * p<0.0001, n=6$ for each of 3 independent experiments

retina was measured from the retinal pigment epithelium to the edge of the nerve-fiber layer. The choroidal layers were measured from the hyper-reflective Bruch's membrane to the choroidal-scleral interface. The outer nuclear layer was measured from the border of the retinal pigment epithelium to the outer plexiform layer interface.

\section{Results}

\section{Concentration-Dependent Inhibition of ATP Release by Tonabersat in the Absence of Pannexin Activity}

The total ATP released from a subconfluent culture of hCMVEC following tonabersat exposure was compared with established membrane channel blockers following $2 \mathrm{~h}$ of ischemia in a model of ischemia injury, or $2 \mathrm{~h}$ after reperfusion (replacement of ischemic media with normal media and ATP levels assayed after a further $2 \mathrm{~h}$ ) (Fig. 1A-C). Connexin43 
A
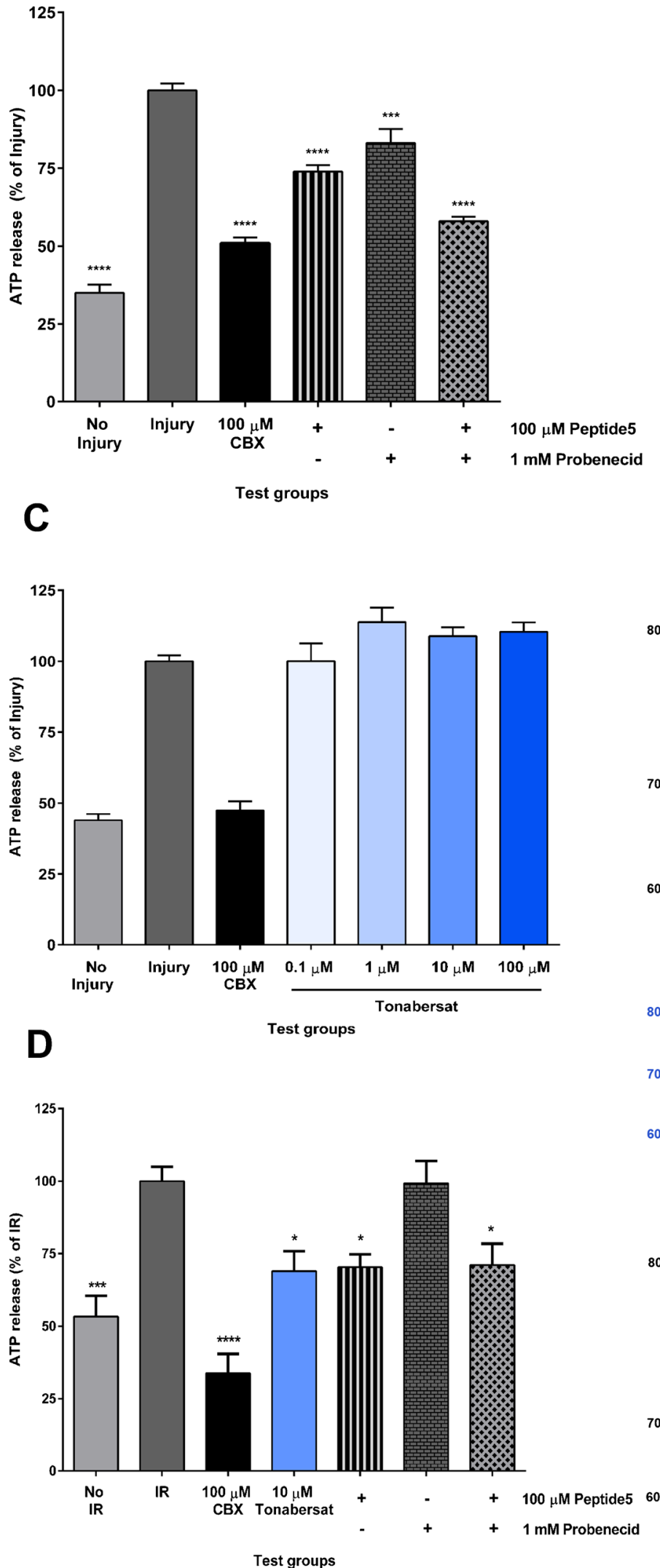

B

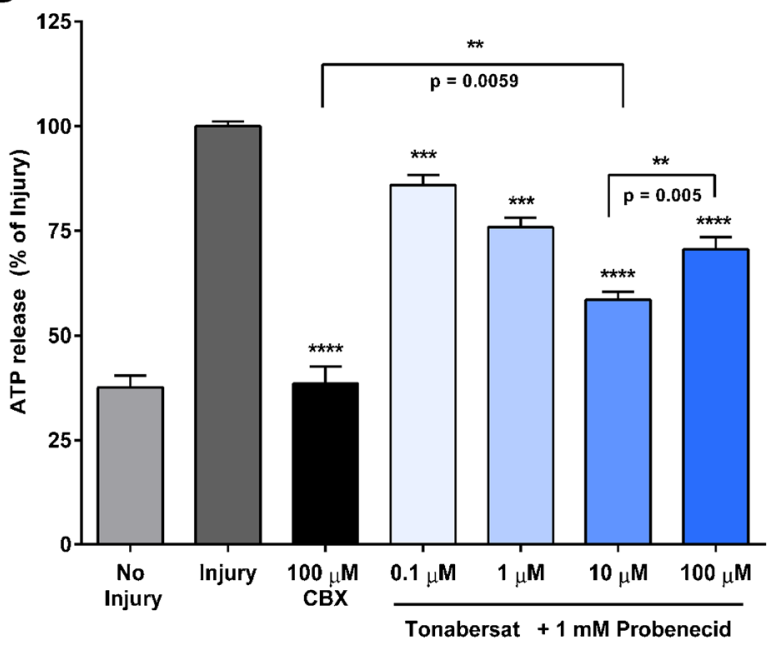

E
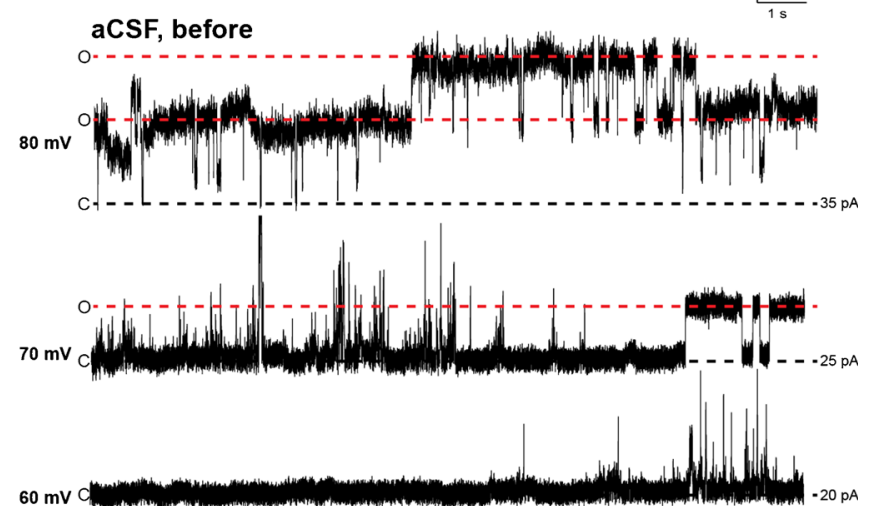

aCSF + $50 \mu \mathrm{M}$ Tonabersat

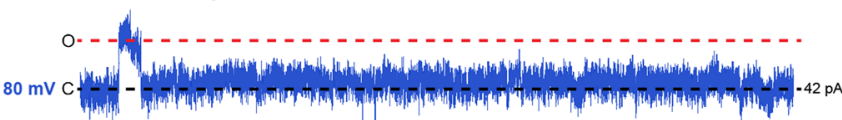

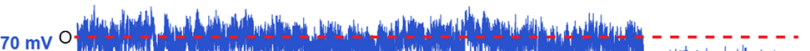

c- -

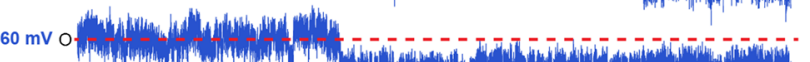

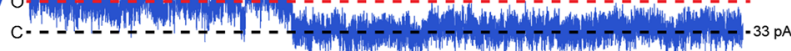
aCSF, washout
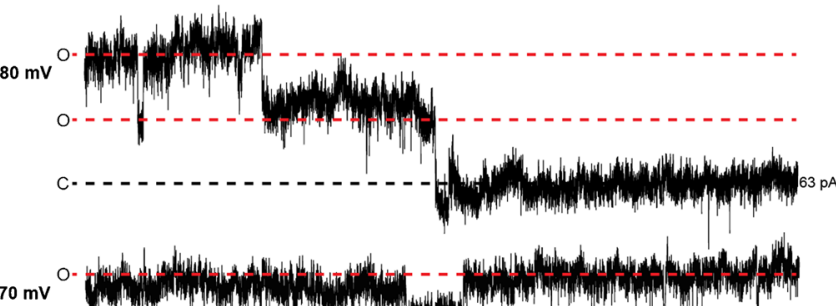

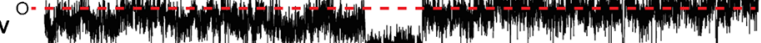

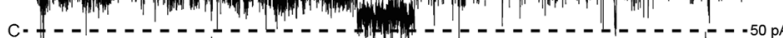

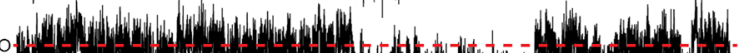

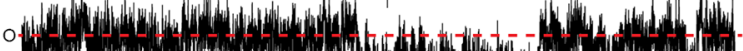

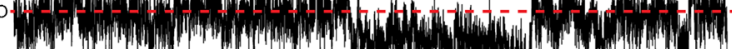

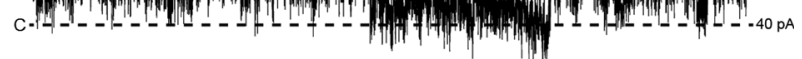


hemichannel- and Pannexin channel-mediated ATP release was differentiated using Peptide5 $(100 \mu \mathrm{M})[56]$ and probenecid (1 mM) [66], respectively. The nonspecific channel blocker CBX $(100 \mu \mathrm{M})$ was used for comparison. ATP standard curves $(0-500 \mathrm{nM}$ concentrations) showed that none of the vehicles or drugs alone affected the assay. Linear regression was found to have an $R^{2}$ of 0.99 for all vehicle and drug combinations.

When compared with the injury group $(100.0 \pm 2.2 \%)$, a basal level of $35.0 \pm 2.6 \%$ ATP was released from the nonischemia control that may reflect normal cellular activity and/or dead cells prior to injury; the difference between these groups was highly significant $(p<0.0001)$ (Fig. 1A). As has been reported previously [71], Peptide5 significantly reduced ATP release to $73.9 \pm 2.1 \%$ during ischemia in hCMVEC and probenecid block of pannexin channels reduced ATP release to $83.1 \% \pm 4.5 \%$. Peptide 5 in combination with probenecid is synergistic and reduced ATP release almost to the same level as CBX [71]. We compared these results with the effect of tonabersat in combination with probenecid (Fig. 1B). ATP release was significantly reduced when probenecid $(1 \mathrm{mM})$ was combined with tonabersat at concentrations of $0.1 \mu \mathrm{M}$ $(85.9 \pm 2.4 \%), 1 \mu \mathrm{M}(75.9 \pm 2.3 \%), 10 \mu \mathrm{M}(58.5 \pm 1.9 \%)$, and $100 \mu \mathrm{M}(70.5 \pm 3.9 \%)$ compared with ischemia injury $(100.0 \pm 1.2 \%$ in this experiment). This effect was concentration dependent- tonabersat at the lowest concentration, 0.1 $\mu \mathrm{M}$, showed no effect over probenecid alone, and maximal inhibition achieved at $10 \mu \mathrm{M}$ tonabersat, and that was significantly greater by $12.1 \pm 3.2 \%$ than the highest concentration of $100 \mu \mathrm{M}$ ( $p=0.005)$ (Fig. 1B). Comparing Figure 1A and $1 \mathrm{~B}$, tonabersat $(10 \mu \mathrm{M})$ was as effective as Peptide5 when probenecid was also present. Interestingly, tonabersat alone under ischaemic conditions did not reduce ATP release from hCMVEC at any of the 4 concentrations used, $0.1,1,10$,or $100 \mu \mathrm{M}(p>0.09)$, although the control channel blocker CBX kept ATP release down to noninjury levels (Fig. 1C).

However, during the reperfusion period, there was no evidence for pannexin channel opening and tonabersat was as effective as Peptide5 whether probenecid was present or not (Fig. 1D). There was a significant difference in ATP release between no injury-reperfusion and injury-reperfusion control $(p=0.0006)$. Baseline ATP release was slightly higher in this experimental group where the medium had been replaced after $2 \mathrm{~h}$ of ischemia in order to mimic reperfusion. Peptide5 significantly reduced ATP release to $70.3 \pm 4.4 \%(p=0.0196)$ under these conditions but probenecid had no effect on ATP release compared with reperfusion injury $(99.3 \pm 4.4 \% ; p>$ 0.99). A combined treatment of Peptide 5 and probenecid also significantly reduced ATP compared with reperfusion injury $(71.1 \pm 7.4 \%, p=0.025)$, which was comparable with Peptide5 treatment alone. Tonabersat $(10 \mu \mathrm{M})$ alone also inhibited ATP release from hCMVEC under these conditions. Indeed, we found a significant reduction in ATP in the presence of $10 \mu \mathrm{M}$ tonabersat $(68.9 \pm 6.9 \% ; p=0.02)$, which was comparable to $100 \mu \mathrm{M}$ Peptide5 during reperfusion injury in vitro (Fig. 1D). As for Peptide5, addition of probenecid with tonabersat had no further effect under reperfusion conditions. CBX $(100 \mu \mathrm{M})$ significantly lowered ATP release to $33.8 \pm 6.6 \%(p=0.0127)$. To confirm that ATP release was from healthy cells, the effect of tonabersat $(0.2-200 \mu \mathrm{M})$ on hCMVEC viability over $24 \mathrm{~h}$ was examined using an MTT assay. Tonabersat $(200 \mu \mathrm{M})$ did not significantly reduce cell viability after $24 \mathrm{~h}$ of treatment $(p=0.378)$. Furthermore, there was no significant reduction in cell viability in response to vehicle $(0.2 \% \mathrm{DMSO})$ or $\mathrm{CBX}(200 \mu \mathrm{M})(p=0.9029$ and $p$ $=0.4719$, respectively).

\section{Tonabersat Reduced Single-Channel Activity in an in vitro Electrophysiology Assay}

We then examined single-channel currents in isolated hCMVEC following stimulation to positive membrane potentials $(\geq 60 \mathrm{mV})$ that are known to activate the opening of connexin 43 hemichannels [25, 32, 72]. A high level of activity was observed at positive membrane potentials in aCSF (Fig. 1E, upper panel). Following perfusion of tonabersat $(50 \mu \mathrm{M})$ in aCSF, an immediate (within 3 min experimental time resolution) reduction in single-channel unitary conductance was apparent (Fig. 1E, middle panel). The single channel unitary currents were partially recoverable following washout of Tonabersat and return to normal aCSF (Fig. 1E, bottom panel). Conductances for $70 \mathrm{mV}$ before, during, and after tonabersat were $45.86 \mathrm{pS}$ [ $95 \%$ confidence interval (CI) 40.69-51.04], 25.1 pS (95\% CI 24.68-25.52) and $38.2 \mathrm{pS}$ (95\% CI 36.9-39.6), respectively.

\section{High Concentrations of Tonabersat Uncoupled GJs in a Time-Dependent Manner}

To test whether tonabersat inhibits GJ communication, we performed an in vitro scrape-loading assay with extracellular LY, a fluorescent dye that is transferred from cell to cell only through coupled GJ [73]. A single scrape wound was made to a confluent monolayer of hCMVEC to enable LY dye to gain access to the cytoplasm of cells at the edge of the wounded region and we then followed transfer of the dye to adjacent cells via GJs. As expected, we observed LY transfer in vehicle control (Fig. 2A), which is indicative of functional cell-to-cell communication via coupled GJs [73]. CBX applied concomitantly with LY significantly reduced GJ communication to $22.2 \pm 2.8 \%$ compared with control $(p<0.0001)$ (Fig. 2B). The extent of GJ uncoupling increased over the tonabersat preincubation time at a set tonabersat concentration of $50 \mu \mathrm{M}$ (Fig. 2A, B). Compared with control, LY-positive cell numbers were $72.5 \pm 7.5 \%$ of controls after preincubation of $50 \mu \mathrm{M}$ tonabersat for $2 \mathrm{~h}(p=0.0132)$ and $62.8 \pm 8.0 \%$ at $6 \mathrm{~h}$ 
A

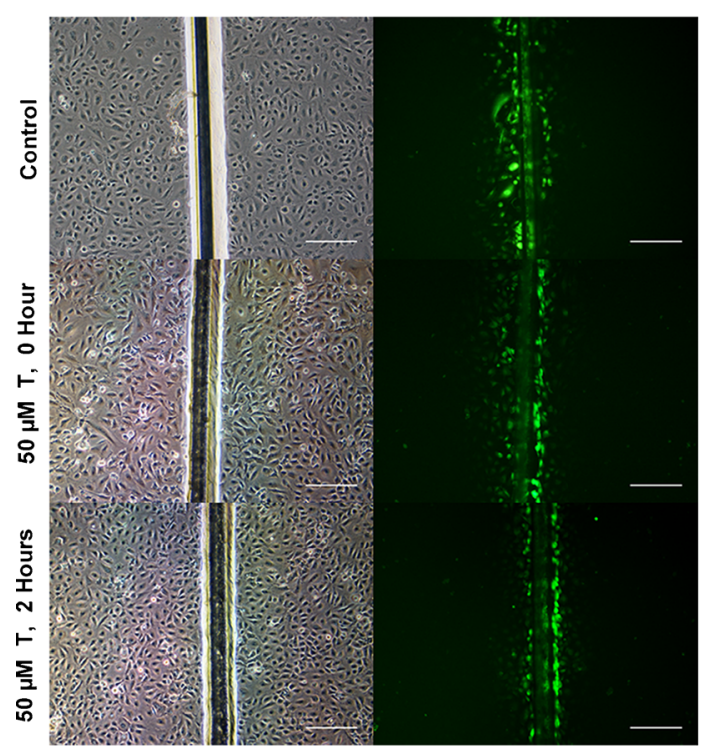

Fig. 2 Functional effect of tonabersat on intercellular communication in human cerebral microvascular endothelial cells (hCMVEC) in vitro. (A) Examples of Lucifer Yellow dye spread to neighboring cells via coupled gap junction as seen after scrape loading. Left-hand panels are phase contrast imaging showing cells and the scrape line; the right-hand panels show the dye uptake and spread. The top panels show control cells, the middle panels show cells treated with $50 \mu \mathrm{M}$ tonabersat for $1 \mathrm{~h}$ prior to scraping, and the bottom panels show cells treated with $50 \mu \mathrm{M}$ tonabersat for $2 \mathrm{~h}$ before scraping. Tonabersat treatment for $1 \mathrm{~h}$ has minimal effect on coupling, but dye spread is reduced after tonabersat treatment for $2 \mathrm{~h}$. (B)
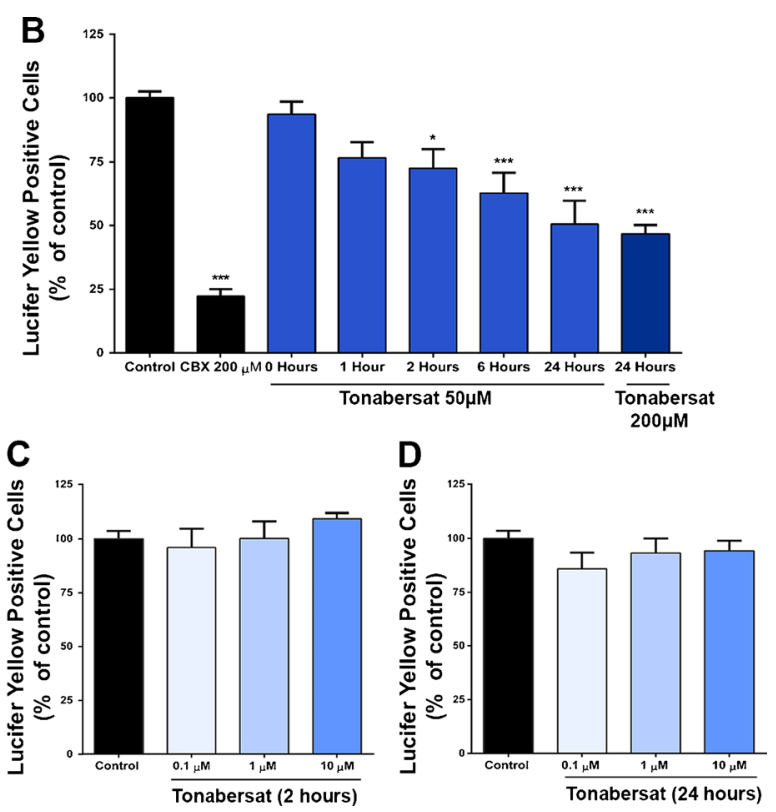

Quantification of dye spread over time with $50 \mu \mathrm{M}$ tonabersat treatment from 0 to $24 \mathrm{~h}$, or at a higher, $200 \mu \mathrm{M}$, concentration for $24 \mathrm{~h}$ before scrape loading. Treatment up to $1 \mathrm{~h}$ before scrape loading did not significantly affect dye spread but the amount of uncoupling (statistically significant) increased with Tonabersat treatment times from 2 to $24 \mathrm{~h}$. (C, D) Low concentrations of tonabersat $(10 \mu \mathrm{M}, 1 \mu \mathrm{M}, 0.1 \mu \mathrm{M})$ did not cause gap junction uncoupling after (C) $2 \mathrm{~h}$ or (D) $24 \mathrm{~h}$. Values represent mean \pm SEM (1-way analysis of variance followed by Tukey's post-hoc test). Scale bars $=200 \mu \mathrm{m} * p<0.05, * * p<0.001, * * * p<0.001 ; n=3$ wells each for each of 3 independent experiments. $\mathrm{CBX}=$ carbenoxolone $(p=0.0003)$. LY-positive cells were maximally reduced to $50.5 \pm 7.5 \%(p<0.0001)$ compared with control by $24 \mathrm{~h}$ (Fig. 2B). After $24 \mathrm{~h}$ preincubation at a higher concentration of $200 \mu \mathrm{M}$ tonabersat, LY-positive cells were further significantly reduced to $46.7 \pm 3.2 \%(p<0.0001)$. However, immediate application of $50 \mu \mathrm{M}$ tonabersat $(p=0.986)$ or $1 \mathrm{~h}$ preincubation prior to the assay $(p=0.054)$ did not significantly reduce GJ coupling when compared with control (Fig. 2B). At lower tonabersat concentrations (0.1, 1, and 10 $\mu \mathrm{M})$, there was no reduction in LY-positive cells at both $2 \mathrm{~h}(p$ $>0.7)$, and $24 \mathrm{~h}(p>0.3)$ of preincubation compared with control (Fig. 2C, D). Taken together, these results indicate that tonabersat-mediated GJ uncoupling is concentrationdependent at $\geq 50 \mu \mathrm{M}$ and the extent of this uncoupling effect is dependent on the time period of tonabersat treatment.

\section{High Concentrations of Tonabersat Caused Internalization and Degradation of Connexin43 Plaques Via the Lysosomal Pathway in ARPE-19 Cells}

We investigated whether the loss of coupling at high tonabersat concentrations was caused by channel block or internalization of connexin43 GJs from the plasma membrane. We used immunocytochemistry in a human cell line, retinal pigmented epithelial cells (ARPE-19), to visualize the distribution and size of the connexin43 GJ plaques. As hCMVEC exhibit interdigitating or overlapping cell-to-cell interfaces they are less suitable for quantifying connexin 43 plaques between cells. We chose ARPE-19 cells because of the classic "crazy paving" pattern that is observed with connexin 43 plaques at clearly defined cell-to-cell borders [74]. We observed connexin43 plaques between cell-to-cell contacts that were labeled in a regular tile-like pattern under basal conditions (Fig. 3A). Connexin43 labeling was also visible in the perinuclear region, which most likely represents an intracellular pool of connexin43 in the Golgi apparatus [75]. However, significant reductions in the total area of connexin 43 were observed following $1 \mathrm{~h}$ of exposure to tonabersat at concentrations of $100 \mu \mathrm{M}(52.8 \pm 5.6 \% ; p=$ $0.0012), 200 \mu \mathrm{M}(63.2 \pm 5.6 \% ; p=0.0286)$, and $500 \mu \mathrm{M}$ $(64.8 \pm 8.4 \% ; p=0.0420)$ compared with untreated control (Fig. 3B). By contrast, compared with untreated control, there was no significant reduction in connexin43 at lower concentrations of $5 \mu \mathrm{M}(p=0.99), 10 \mu \mathrm{M}(p=0.5777)$, or $50 \mu \mathrm{M}(p$ $=0.1146)($ Fig. 3B).

Next, we investigated whether the reduction in connexin 43 plaques was time-dependent by exposing ARPE-19 cells to tonabersat $(50-500 \mu \mathrm{M})$ for an extended period of $6 \mathrm{~h}$ 
A

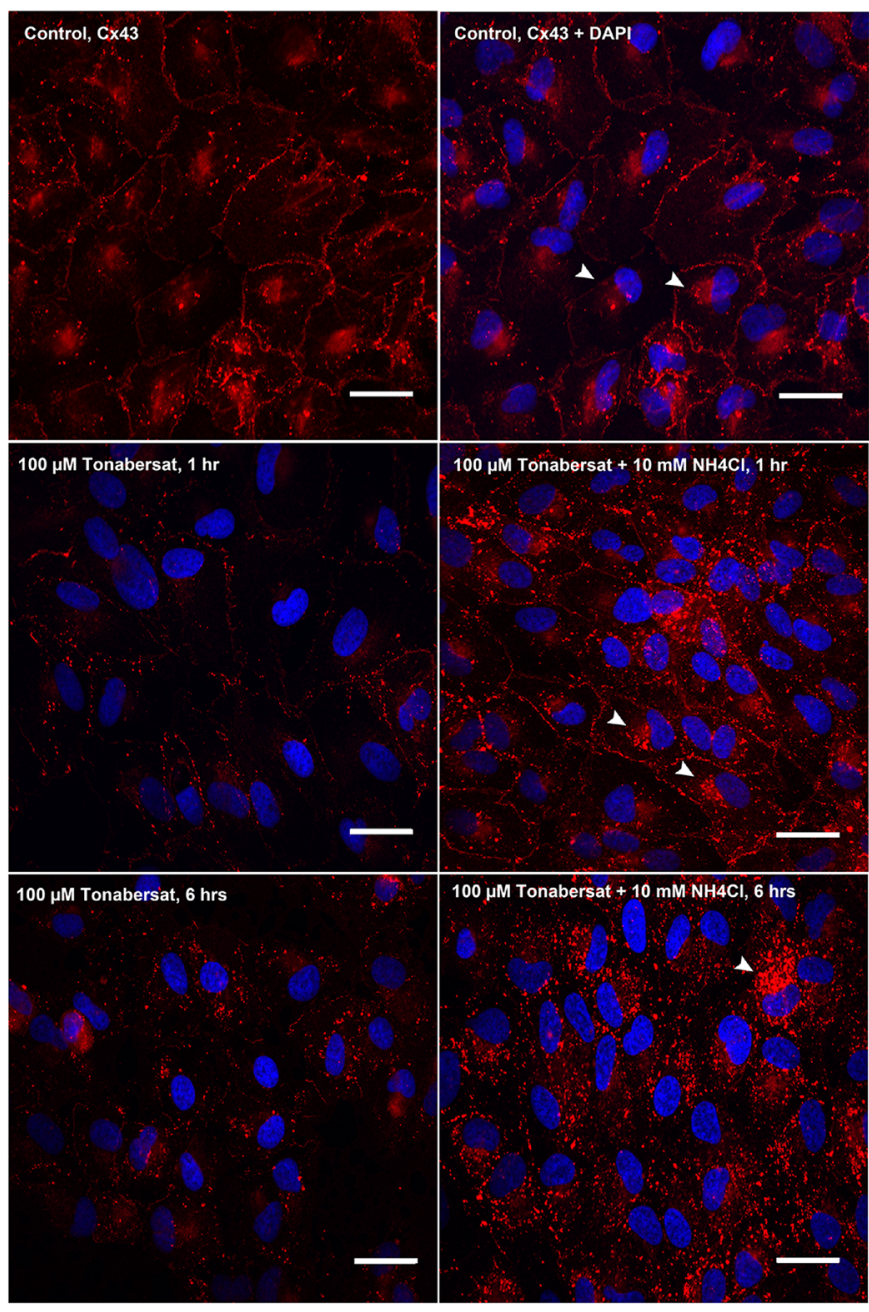

B
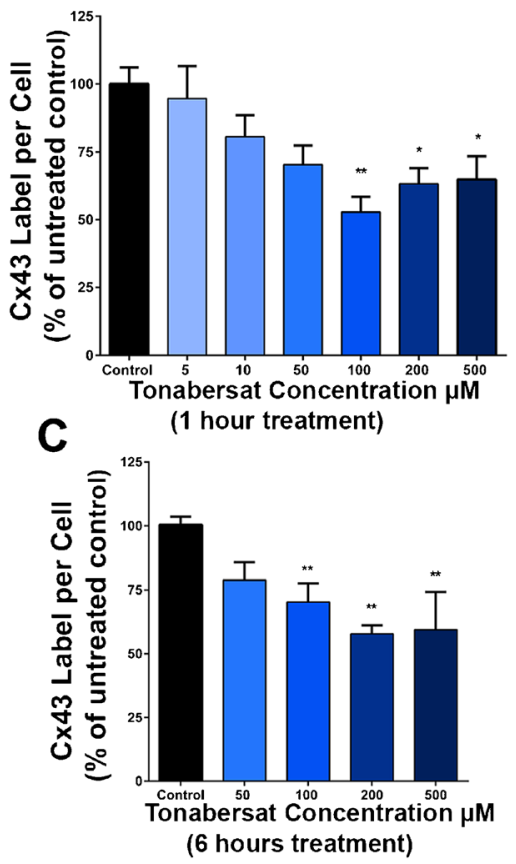

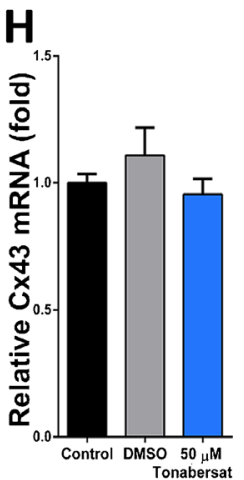

$\mathbf{E}$

G
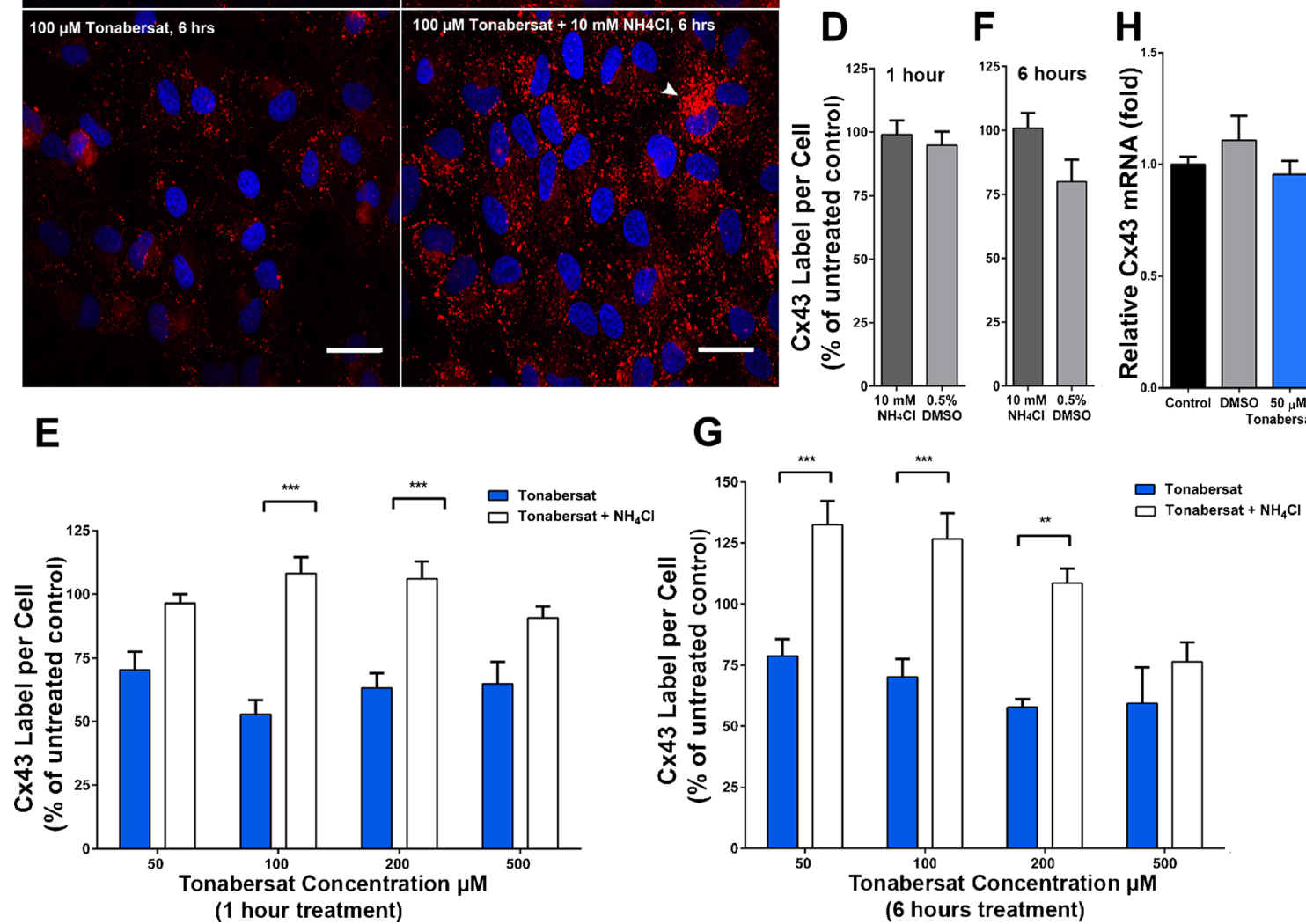

(Fig. 3C), which also led to reductions in connexin43 labeling between cell-to-cell contacts and a reduction in the intracellular pool (Fig. 3A). The total area of connexin43 labeling was significantly reduced at $100 \mu \mathrm{M}(66.5 \pm 5.4 \% ; p<0.001)$, $200 \mu \mathrm{M}(62.5 \pm 2.3 \% ; p<0.001)$, and $500 \mu \mathrm{M}(60.2 \pm 13.6 \%$; $p<0.001$ ) (Fig. 3C).

We then proposed that tonabersat at higher doses may facilitate turnover of connexin 43 plaques from the plasma membrane. To test this, we immobilized the lysosomal degradation pathway in ARPE-19 cells using ammonium chloride $\left(\mathrm{NH}_{4} \mathrm{Cl}\right)$, a weak base lysosome inhibitor [68]. Following 1-h treatment in 50 to $500 \mu \mathrm{M}$ tonabersat and $10 \mathrm{mM} \mathrm{NH}_{4} \mathrm{Cl}$, 
Fig. 3 Tonabersat internalizes and degrades connexin43 gap junction plaques via the lysosomal pathway in ARPE-19 cells. (A) Immunolabeling for connexin43 (Cx43; red) and 4',6-diamidino-2phenylindole (DAPI)-labeled nuclei (blue) in untreated control cells (upper panel), after $100 \mu \mathrm{M}$ tonabersat for $1 \mathrm{~h}$ (middle left), $100 \mu \mathrm{M}$ tonabersat $+\mathrm{NH}_{4} \mathrm{Cl}$ for $1 \mathrm{~h}$ in serum-supplemented media (middle right), $100 \mu \mathrm{M}$ tonabersat for $6 \mathrm{~h}$ (lower left), or $100 \mu \mathrm{M}$ tonabersat $+\mathrm{NH}_{4} \mathrm{Cl}$ for $6 \mathrm{~h}$ (lower right). White arrows indicate perinuclear labeling of connexin43; scale bars $=30 \mu \mathrm{m}$. Quantification of the total area of connexin 43 plaques per cell following treatment with tonabersat (5-500 $\mu \mathrm{M})$ for $1 \mathrm{~h}$ is seen in (B), and tonabersat $(50-500 \mu \mathrm{M})$ for $6 \mathrm{~h}$ in $(\mathrm{C})$. (B, C) Normalized to untreated control and expressed as a percentage. Quantification of the total area of connexin 43 plaques per cell following treatment with tonabersat $(50-500 \mu \mathrm{M})$ with $\mathrm{NH}_{4} \mathrm{Cl}$ for $1 \mathrm{~h}$ is seen in (E) and for $6 \mathrm{~h}$ in $(\mathrm{G})$ with both figures normaliszed to untreated control and expressed as a percentage. $\mathrm{NH}_{4} \mathrm{Cl}$ prevents junctional degradation, indicating that it would otherwise occur via the lysosomal pathway. No significant difference in connexin43 labeling was present between untreated control and $10 \mathrm{mM} \mathrm{NH} 4 \mathrm{Cl}$ or vehicle control [0.5\% dimethyl sulfoxide (DMSO)] alone for (D) $1 \mathrm{~h}$ or (E) $6 \mathrm{~h}$. Confluent monolayers of ARPE-19 cells were incubated with DMSO (vehicle control), or with $50 \mu \mathrm{M}$ tonabersat for $1 \mathrm{~h}$ and relative connexin 43 mRNA levels were then assessed. (H) Tonabersat has no effect on connexin43 transcription. Values represent mean \pm SEM. One-way analysis of variance followed by Tukey's multiple comparisons test. *p $<0.05$; * $p<0.01 * * * p<$ $0.001 ; n=3$ wells per treatment for each of 2 independent experiments

connexin43 plaques were removed from cell-to-cell contacts but remained in the cytoplasm with punctate labeling along the intracellular region immediately below the plasma membrane (Fig. 3A). In addition, there was now dense labeling visible around the perinucleus (in what appears to be the Golgi body) (Fig. 3A, white arrows). Treatment with $\mathrm{NH}_{4} \mathrm{Cl}$ for $1 \mathrm{~h}$, or the vehicle control $(0.5 \%$ DMSO) on its own, had no significant effect on connexin43 labeling per cell compared with the untreated control $(p>0.8)$ (Fig. 3D). However, following treatment with tonabersat in the presence of $10 \mathrm{mM} \mathrm{NH}_{4} \mathrm{Cl}$, total connexin43 labeling was significantly increased by $104.8 \%(p<0.001)$ and $65.4 \%(p=0.0002)$ compared with $100 \mu \mathrm{M}$ and $200 \mu \mathrm{M}$ tonabersat concentrations alone, respectively (Fig. 3E).

Following $6 \mathrm{~h}$ of treatment in tonabersat and $10 \mathrm{mM} \mathrm{NH}_{4} \mathrm{Cl}$, punctate connexin43 labeling was distributed throughout the cell (Fig. 3A). In contrast to the 1-h treatment period, there was no visible connexin43 labeling between cell-to-cell contacts (Fig. 3A). Treatment with $\mathrm{NH}_{4} \mathrm{Cl}$ for $6 \mathrm{~h}$, or $0.5 \%$ DMSO vehicle control on its own, had no significant effect on the total area of connexin43 labeling per cell compared with untreated control $(p>0.06)$ (Fig. 3F). However, 6-h treatment with Tonabersat in the presence of $10 \mathrm{mM} \mathrm{NH}_{4} \mathrm{Cl}$ significantly increased the total amount of connexin43 labeling by $67.9 \%$ ( $p=$ $0.0015), 80.3 \%(p=0.0001)$, and $87.9 \%(p=0.0062)$ compared with 50,100 , and $200 \mu \mathrm{M}$ tonabersat treatment alone, respectively (Fig. 3G).

To confirm that tonabersat-mediated reduction in connexin43 plaques seen in Figure 3(A) was caused by internalization, and not a downregulation in connexin43 mRNA transcription, we used real-time reverse transcriptase PCR to compare the relative differences in connexin 43 mRNA following a 1-h treatment with tonabersat. There was no significant difference in connexin43 mRNA in ARPE-19 cells following treatment with $50 \mu \mathrm{M}$ tonabersat compared with both untreated $(p=0.7572)$ and vehicle controls $(p=0.10245)$ (Fig. 3H).

\section{Tonabersat Prevented Loss of Retinal Function and Preserved Retinal Integrity After Retina Bright-Light Damage in a Rat Model}

The effect of a connexin hemichannel block in this model has been described previously using the mimetic peptide hemichannel blocker Peptide5 [50]. For comparison, the effect of tonabersat in the retina light-damage rat model was tested using ERG analysis and OCT. The ERG was conducted 24 h, 1 week, and 2 weeks following intense light exposure. Representative mixed ERG waveforms of sham and tonabersat-treated rats at 2 weeks after light damage are shown in Figure 4. Overall, mixed a-wave amplitude was better preserved in tonabersat-treated rats as a function of time compared with sham-treated rats at intensities of -1.9 to $2.1 \mathrm{log}$ cd.s $/ \mathrm{m}^{2}$. There was a significant difference in the a-wave amplitude $24 \mathrm{~h}$ post-treatment at 1.6 to $1.8 \mathrm{log} \mathrm{cd} . \mathrm{s} / \mathrm{m}^{2}(p<0.05)$ and 1 week at 0.1 to $2.1 \mathrm{log} \mathrm{cd} . \mathrm{s} / \mathrm{m}^{2}(p<0.01-0.0001)$ compared with the sham group (Fig. 4A). At 2 weeks post-treatment, retinal function was significantly preserved compared with sham-treated animals between 0.1 and $2.1 \log \mathrm{cd} . \mathrm{s} / \mathrm{m}^{2}(p$ $<0.01$ and $p<0.0001$, respectively).

Further analysis showed that the mixed b-wave amplitude of the ERG was also better preserved as a function of time post-treatment. The mixed b-wave that reflects the inner retina function was preserved in tonabersat-treated rats as a function of time compared with sham rats at intensities -3.9 to $2.1 \mathrm{log}$ cd.s $/ \mathrm{m}^{2}$ where a significant decline in retinal function was observed as a result of injury. There was a significant difference in b-wave amplitude $24 \mathrm{~h}$ post-treatment at -2.9 and -2.1 $\log$ cd.s $/ \mathrm{m}^{2}(p<0.05$ to $p<0.01), 1$ week post-treatment at 1.6 to $2.1 \log \mathrm{cd} . \mathrm{s} / \mathrm{m}^{2}(p<0.01$ to $p<0.001)$, and 2 weeks following intense light damage at 3.9 to $2.1 \mathrm{log} \mathrm{cd} . \mathrm{s} / \mathrm{m}^{2}$ compared with the sham-treated group (Fig. 4B; $p<0.01-0.001$.).

The isolated rod PIII and PII amplitudes which were extracted at the highest flash intensity, $2.1 \log \mathrm{cd} . \mathrm{s} / \mathrm{m}^{2}$, also exhibited a progressive recovery of the rod photoreceptoral activity when the tonabersat-treated group was compared with the sham-treated LD group. There was a significant positive correlation in PIII amplitude between 1 week and 2 weeks post-treatment of tonabersat compared with the sham-treated group ( $p<0.01$ and $p<0.001$ ) (Fig. 4C). For the rod PII amplitude, there was a significant difference at 2 weeks post-tonabersat treatment compared with the sham-treated group $(p<0.05)$ (Fig. 4D). Interestingly, the cone PII 


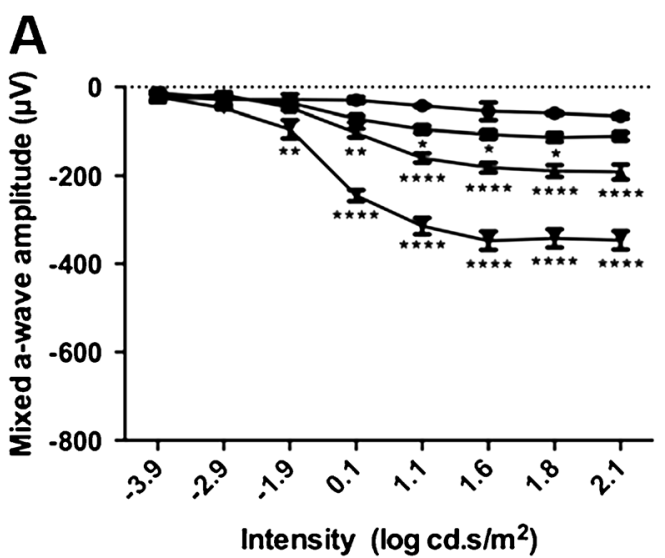

C
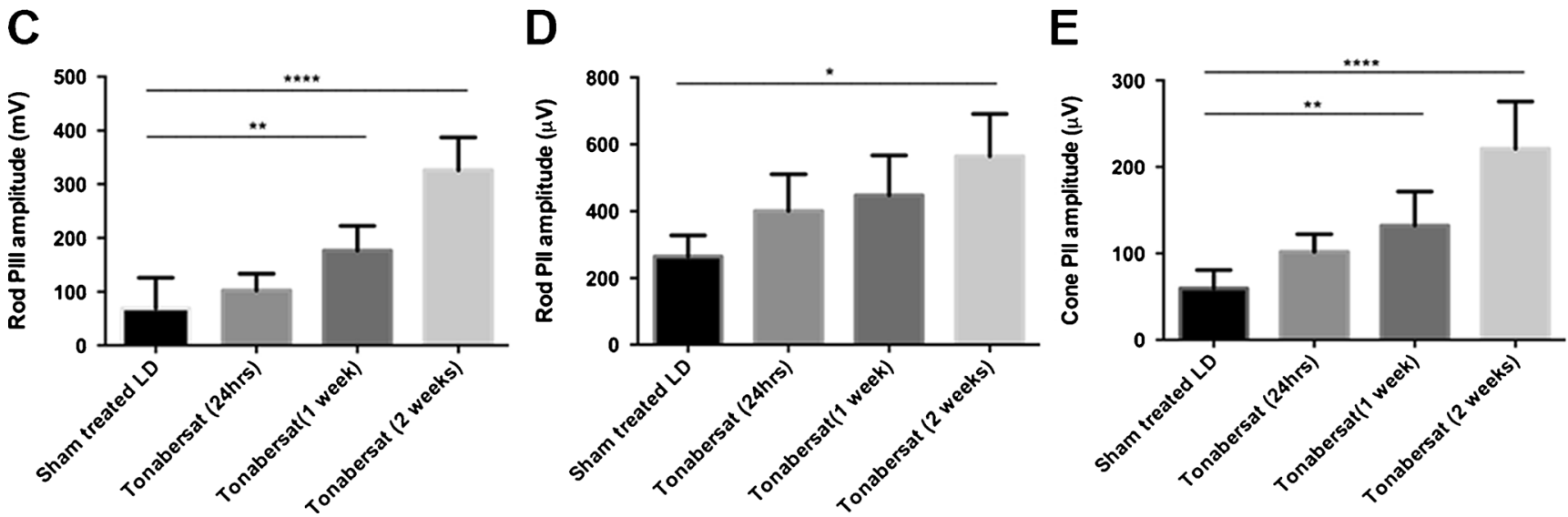

Fig. 4 Tonabersat treatment reduces the loss of retinal function in the bright-light damage model ( 6 animals per group, 12 eyes analyzed). Representative electroretinogram (ERG) waveforms for light intensities ranging from -3.9 to $2.1 \mathrm{log} \mathrm{cd} . \mathrm{s} / \mathrm{m}^{2}$ of sham-treated ?? (LD) and tonabersat-treated animals in a light-damaged rat retina model. Tonabersat leads to significantly improved a-wave amplitude compared

amplitude observed at the highest flash intensity, $2.1 \log \mathrm{cd} . \mathrm{s} /$ $\mathrm{m}^{2}$, was improved at both 1 week and 2 weeks post-light damage with statistically significant differences $(p<0.01$ and $p<0.001$, respectively) (Fig. 4E).

The sparing effect of tonabersat on the retina after brightlight damage was also investigated using OCT. Figure 5 shows in vivo imaging of the retinal and choroidal layers in the sham rats and tonabersat-treated rats. Retinal layers were thinner than the average normal SD rat retina in the shamtreated, light-damaged rat eyes, although there were no significant fundus changes observed over the 2 -week period (Fig. 5A). There was a significant thinning of the outer nuclear layer $(\mathrm{ONL})$ in the postinjury sham-treated group compared with the thickness of this layer prior to injury $(p<0.001$; Fig. 5C). In contrast, there was no significant loss in ONL thickness in the tonabersat-treated group at any time point compared with retinal thickness prior to the injury. There was also a significant loss in choroid thickness in the shamtreated group compared with the choroid thickness prior to injury ( $p<0.001$; Fig. 5D). As for the ONL there was no with (A) sham-treated and (B) b-wave amplitude compared with shamtreated (assessed at 2 weeks). (C) Rod PIII amplitude, (D) rod PII amplitude, and cone PII amplitude in the ERG analysis are all improved after tonabersat treatment compared with sham treatment, with improvement increasing over time. $* p<0.05$, ** $p<0.01, * * * p<0.001$, **** $p<$ 0.0001

decrease in choroid thickness in the tonabersat-treated group compared with choroid thickness prior to the injury.

\section{Discussion}

A major finding of this study is that tonabersat significantly inhibits connexin hemichannel opening. Exposure to high concentrations of tonabersat in vitro $(>100 \mu \mathrm{M})$ uncoupled GJs with sustained long-term treatment causing connexin 43 plaques to be targeted for internalization and degradation via the lysosomal pathway. Even at a concentration of $200 \mu \mathrm{M}$, however, about $50 \%$ of functional GJ communication, assessed using the LY scrape-loading assay, was retained following prolonged 24-h exposure. While immunohistochemistry indicated internalization of connexin 43 plaques with tonabersat treatment, based on data from the scrape-loading assay it appears that a number of functional GJ channels remain dispersed within the membrane. In contrast, exposure to low concentrations of tonabersat $(10 \mu \mathrm{M})$ effectively inhibited 

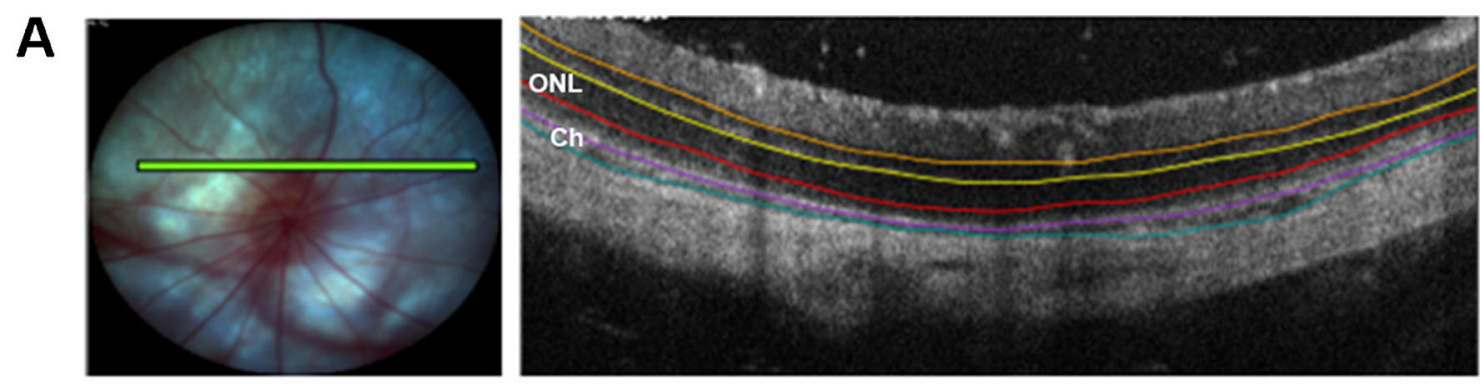

B
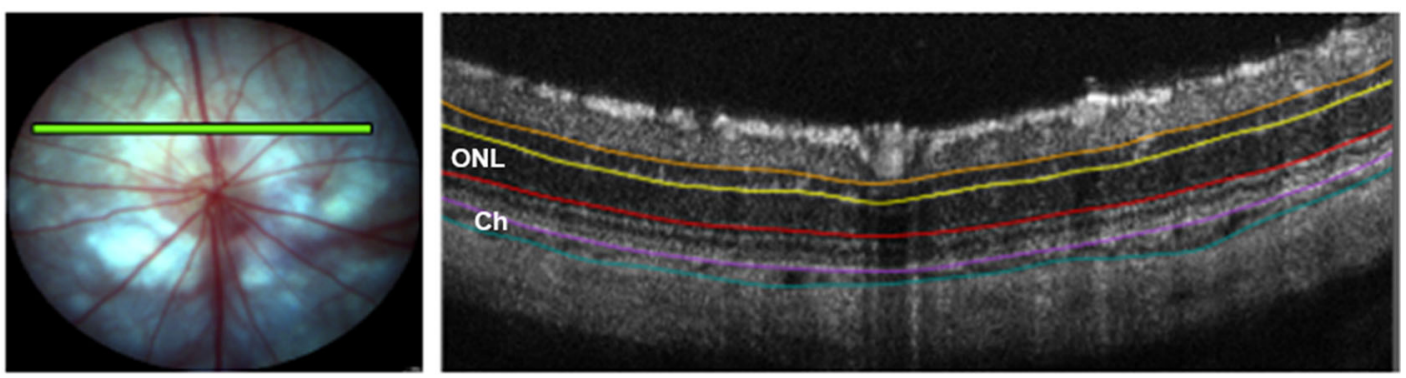

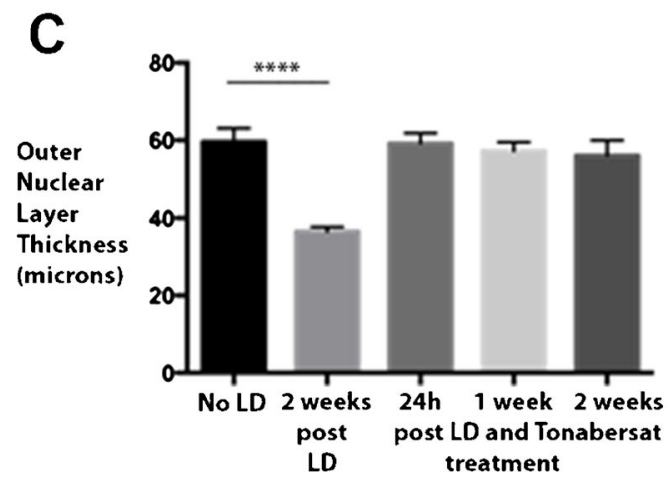

Fig. 5 Tonabersat treatment in the light-damaged rat model prevents the loss of both retinal and choroidal thickness. Representative fundus and optical coherence tomography (OCT) images of sham-treated animal retina are seen in (A) and tonabersat-treated animal retina in (B) 2 weeks postinjury and treatment. A green scanning line on the fundus image represents the exact location of the cross-sectional OCT image $(n=6$

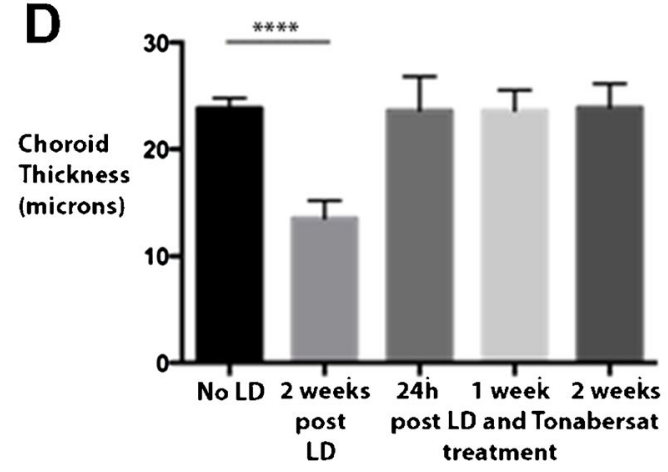

per group). Both the outer nucleus layer and choroid thickness is reduced in the injured but sham-treated animals, but retinal thickness was preserved with tonabersat treatment. The thickness of the (C) choroid and the (D) outer nuclear layer are expressed as mean $\pm \mathrm{SD}$. $* * * * p<0.0001$ $\mathrm{LD}=$ ??; $\mathrm{ONL}=$ outer nuclear layer $; \mathrm{Ch}=$ choroid

amount of ATP released into the extracellular space as a function of channel activity. As pannexin channels can also permeate ATP in endothelial cells [79], and have also been proposed to open under injury conditions [80], connexin mimetic peptide, Peptide5, at $100 \mu \mathrm{M}$ concentration [56] and $1 \mathrm{mM}$ probenecid [66] were used to establish the model and to discriminate connexin43 hemichannel and pannexin channel function, respectively. During ischemia, ATP release from connexin hemichannels was seen to be 1.5 times that released from pannexin channels and the combination of Peptide5 and probenecid reduced the level of ATP release to near that of the nonspecific channel blocker CBX.

Using the in vitro injury model described above then, we have shown a concentration-dependent inhibition of connexin hemichannels by tonabersat. A low tonabersat $(10 \mu \mathrm{M})$ concentration was the most effective at inhibiting hemichannel opening, and it was surprising that $100 \mu \mathrm{M}$ tonabersat was 
not as effective in lowering ATP release as $10 \mu \mathrm{M}$ tonabersat. As higher tonabersat concentrations were not linked to cell toxicity, it is possible that tonabersat may have dual effects during ischemic injury whereby connexin hemichannels are targeted for inhibition, but pannexin channels may be triggered to open in an off-target effect. While further research is required to confirm any action of tonabersat on pannexin channels, our present data suggest that a low concentration of tonabersat combined with a pannexin channel blocker, such as probenecid, may be most effective during ischemic injury in vitro.

Ischemia is not a single event, and cellular injury to peritraumatic regions is attributed to lesion spread postischemia [81]. Connexin43 hemichannels have been shown to be upregulated 4 to $8 \mathrm{~h}$ after injury in several in vivo models [37, 43, 46, 53, 54], and this has been linked to cell swelling [82, 83], vessel leak, inflammation, and cell death $[16,37,84]$. As discussed above, connexin hemichannels open in response to conditions that are typical of ischemia, but whether hemichannels open both during and after the initial ischemic injury has been debated. Therefore, we also created post-ischaemia-reperfusion conditions in vitro by returning to normal physiologic conditions after $2 \mathrm{~h}$ in HAIR solution. Postischemia, connexin hemichannels accounted for almost all ATP release (Fig. 1D). Importantly, tonabersat at $10 \mu \mathrm{M}$ concentration was shown to be comparable to $100 \mu \mathrm{M}$ of Peptide5 in inhibiting ATP release from hCMVEC. These data suggest that a low concentration of tonabersat alone can inhibit connexin hemichannel-mediated ATP release postischemia.

Tonabersat-mediated inhibition of ATP release through connexin 43 hemichannels could have implications for attenuating inflammation and inflammasome activation postinjury (for review see [40, 84]). Intracellular ATP is crucial for energy transfer [85], but a rapid increase of extracellular ATP upon ischemia [86], spinal cord injury [42], or epilepsy-initiated seizures [87] can activate ATP-gated receptors (i.e., $\mathrm{P}_{2} \mathrm{X}$ receptors) expressed on the surface of astrocytes and microglia [88], leukocytes [89], and endothelial cells [90]. This event is associated, in particular, with the activation of the NLR pyrin domain containing 3 inflammasome protein complex, a major component of the innate immune system [91]. The NLR pyrin domain containing 3 inflammasome protein complex promotes the maturation and release of proinflammatory cytokines, such as IL-1 $\beta$ and IL-18 $[92,93]$. These proinflammatory cytokines can induce secondary damage at the systemic level (sepsis) [94], as well as local lesion spread in the peritraumatic region, including proliferation of astrocytes (astrogliosis) [95].

In an in vivo bright light-induced retina damage model, the inflammatory response starts in the choroid before leading to oxidative stress in the retina, and those changes are associated with increased connexin 43 expression [54]. Loss of retinal function can be attributed to open connexin 43 hemichannels as a low dose of connexin 43 mimetic Peptide5 that targets connexin43 hemichannels but not GJ [56], significantly improved functional outcomes [50]. Better functional outcomes for neurons in both the rod and cone phototransduction pathways was likely owing to reduced choroid inflammation and a suppressed glial-mediated inflammatory response bought about by hemichannel block. We have applied the same in vivo model used by [50] to show that tonabersat may also be protective against functional loss following retinal brightlight damage, reported to be a model for the dry form of agerelated macular degeneration [96]. Tonabersat was delivered by intraperitoneal injection to provide a final circulating concentration of $10 \mu \mathrm{M}$ (assuming total uptake and a $20 \mathrm{ml}$ blood volume), selected to match the optimal concentration in the in vitro ATP release assay.

In most phase II clinical trials that involve tonabersat for the treatment of migraine, it has been used at a daily dosage of 20 to $80 \mathrm{mg}$ [7]. Tonabersat has also been proposed as a treatment for epilepsy [97, 98]. Our study adds to previous work where a single dose of tonabersat $(10 \mathrm{mg} / \mathrm{kg})$ was reported to counteract the proinflammatory mediator-induced upregulation of connexin $26 \mathrm{GJ}$ expression between trigeminal neurons and satellite glial cells, and was attributed to affecting the p38-MAPK pathway in the CNS [12]. From our current understanding, there is no evidence showing that tonabersat is specific to connexin 26 channels or the p38-MAPK pathway. Whilst tonabersat has been proposed to bind with high affinity to a stereoselective, yet unspecified site in the CNS of human and rat [1] it may also not be CNS-specific. The dose used in most previous animal studies $(10 \mathrm{mg} / \mathrm{kg})$ is significantly higher than that used in our study $(0.32 \mathrm{mg} / \mathrm{kg})$. We showed that high concentrations of tonabersat induced connexin $43 \mathrm{GJ}$ internalization with protein sent for degradation via the lysosomal pathway, although about $50 \%$ of functional GJ coupling was retained even following prolonged 24-h exposure to tonabersat at a high concentration of $200 \mu \mathrm{M}$ in vitro. Surprisingly, an increase in labeling in the $\mathrm{NH}_{4} \mathrm{Cl}$ control-treated cells was not observed, which may reflect a restriction in labeling of connexin protein within endocytosed vesicles (steric hindrance or vesicle permeabilization) using our immunohistochemistry protocol. Both our in vitro and in vivo data support the idea that tonabersat may be acting primarily as a connexin 43 hemichannel blocker, with any upregulation previously reported for connexin 26 expression possibly a downstream consequence of reduced inflammation.

Connexin 43 expression is often elevated after injury, as seen in spinal cord injury [55, 99], CNS ischemia [37, $100,101]$, retinal injury [50], epilepsy [102], and in chronic disease such as Huntington's disease [103]. This is not always the case (for review see [84]), but it is also increasingly evident that hemichannel opening probability may increase under injury and disease conditions, where cell-to-cell coupling is reduced [27, 30, 104-108]. 
In conclusion, we provide evidence for novel mechanisms of action of tonabersat that include a direct and immediate block of connexin hemichannels, and, at higher concentrations, a concentration- and time-dependent inhibition of connexin 43 GJ coupling. Both connexin hemichannels and pannexin channels mediate ATP release during ischemic injury in microvascular endothelial cells, but connexin hemichannels appear to be the primary contributor to ATP leak postinjury. Short-term exposure to low concentrations of tonabersat effectively inhibited connexin 43 hemichannel-mediated ATP release during injury but especially during reperfusion in vitro, and this was consistent with reduced loss of retinal function and integrity in an in vivo rat bright-light injury model of retinal damage. We conclude, therefore, that tonabersat is not glia neuron-specific as previously suggested, is not restricted to connexin26 expression modulation, and does not affect connexin43 protein synthesis. Instead, tonabersat appears to be acting primarily and directly on connexin hemichannels to reduce channel opening under injury conditions. Tonabersat may offer an orally available approach for various injuries and chronic inflammatory disease indications, including muscular dystrophy [109], amyotrophic lateral sclerosis [110], CNS trauma [43, 44], stroke or ischemia [46, 47, 111, 112], glaucoma, diabetic retinopathy and macular degeneration $[37,49,50,54,113]$, as well as Alzheimer's disease [110, 114], chronic pain [115], and infectious disease [116] where connexin hemichannels are implicated in injury spread and the perpetuation of inflammatory processes, especially through the inflammasome pathway [40, 84, 109].

Acknowledgements We thank Professor P.J. Donaldson for the generous loan of his electrophysiology equipment. This work was supported by a University of Auckland Doctoral Scholarship to Y.K. J.M.G was supported by the CatWalk Spinal Cord Injury Trust, and M.N.M.N by a PhD scholarship from the Ministry of Higher Education, Malaysia. C.R.G is a founding scientist of CoDa Therapeutics, Inc. USA, which holds intellectual property around the use of connexin channel modulators for the treatment of injury and disease. C.R.G and Y.K are named as inventors on patent application(s) related to uses of tonabersat. We acknowledge support from Auckland UniServices Ltd and the New Zealand Return on Science fund. C.R.G is the W\&B Hadden Professor of Ophthalmology and Translational Research and acknowledges the generous support of W\& B Hadden.

Required Author Forms Disclosure forms provided by the authors are available with the online version of this article.

\section{References}

1. Chan WN, Evans JM, Hadley MS, et al. Identification of (-)-cis-6acetyl-4S-(3-chloro-4-fluoro-benzoylamino)- 3,4-dihydro-2,2-dimethyl-2H-benzo[b]pyran-3S-ol as a potential antimigraine agent. Bioorganic \& medicinal chemistry letters. 1999;9:285-90.

2. Read SJ, Smith MI, Hunter AJ, Upton N, Parsons AA. SB220453, a potential novel antimigraine agent, inhibits nitric oxide release following induction of cortical spreading depression in the anaesthetized cat. Cephalalgia. 2000;20:92-9.
3. Durham PL, Garrett FG. Neurological mechanisms of migraine: potential of the gap-junction modulator tonabersat in prevention of migraine. Cephalalgia. 2009;29 Suppl 2:1-6.

4. Dohmen C, Sakowitz OW, Fabricius M, et al. Spreading depolarizations occur in human ischemic stroke with high incidence. Annals of neurology. 2008;63:720-8.

5. Dreier JP, Major S, Pannek HW, et al. Spreading convulsions, spreading depolarization and epileptogenesis in human cerebral cortex. Brain : a journal of neurology. 2012;135:259-75.

6. Fabricius M, Fuhr S, Bhatia R, et al. Cortical spreading depression and peri-infarct depolarization in acutely injured human cerebral cortex. Brain : a journal of neurology. 2006;129:778-90.

7. Hauge AW, Asghar MS, Schytz HW, Christensen K, Olesen J. Effects of tonabersat on migraine with aura: a randomised, double-blind, placebo-controlled crossover study. The Lancet Neurology. 2009;8:718-23.

8. Goadsby PJ, Ferrari MD, Csanyi A, et al. Randomized, doubleblind, placebo-controlled, proof-of-concept study of the cortical spreading depression inhibiting agent tonabersat in migraine prophylaxis. Cephalalgia. 2009;29:742-50.

9. MaassenVanDenBrink A, van den Broek RW, de Vries R, et al. The potential anti-migraine compound SB-220453 does not contract human isolated blood vessels or myocardium; a comparison with sumatriptan. Cephalalgia. 2000;20:538-45.

10. Bialer M, Johannessen SI, Levy RH, et al. Progress report on new antiepileptic drugs: a summary of the Eleventh Eilat Conference (EILAT XI). Epilepsy research. 2013;103:2-30.

11. Dahlof CG, Hauge AW, Olesen J. Efficacy and safety of tonabersat, a gap-junction modulator, in the acute treatment of migraine: a double-blind, parallel-group, randomized study. Cephalalgia. 2009;29 Suppl 2:7-16.

12. Damodaram S, Thalakoti S, Freeman SE, Garrett FG, Durham PL. Tonabersat inhibits trigeminal ganglion neuronal-satellite glial cell signaling. Headache. 2009;49:5-20.

13. Dermietzel R, Hertberg EL, Kessler JA, Spray DC. Gap junctions between cultured astrocytes: immunocytochemical, molecular, and electrophysiological analysis. The Journal of neuroscience : the official journal of the Society for Neuroscience. 1991;11:142132.

14. Dermietzel R, Traub O, Hwang TK, et al. Differential expression of three gap junction proteins in developing and mature brain tissues. Proceedings of the National Academy of Sciences of the United States of America. 1989;86:10148-52.

15. Nagy JI, Yamamoto T, Sawchuk MA, Nance DM, Hertzberg EL. Quantitative immunohistochemical and biochemical correlates of connexin43 localization in rat brain. Glia. 1992;5:1-9.

16. Decrock E, De Bock M, Wang N, et al. Connexin and pannexin signaling pathways, an architectural blueprint for CNS physiology and pathology? Cell Mol Life Sci. 2015;72:2823-51.

17. Zimmer DB, Green CR, Evans WH, Gilula NB. Topological analysis of the major protein in isolated intact rat liver gap junctions and gap junction-derived single membrane structures. The Journal of biological chemistry. 1987;262:7751-63.

18. Makowski L, Caspar DL, Phillips WC, Goodenough DA. Gap junction structures. II. Analysis of the x-ray diffraction data. The Journal of cell biology. 1977;74:629-45.

19. Li H, Liu TF, Lazrak A, et al. Properties and regulation of gap junctional hemichannels in the plasma membranes of cultured cells. The Journal of cell biology. 1996;134:1019-30.

20. Simpson I, Rose B, Loewenstein WR. Size limit of molecules permeating the junctional membrane channels. Science. 1977;195:294-6.

21. Harris AL. Connexin channel permeability to cytoplasmic molecules. Progress in biophysics and molecular biology. 2007;94:120-43.

22. Chever O, Lee CY, Rouach N. Astroglial connexin 43 hemichannels tune basal excitatory synaptic transmission. The Journal of 
neuroscience : the official journal of the Society for Neuroscience. 2014;34:11228-32.

23. Stehberg J, Moraga-Amaro R, Salazar C, et al. Release of gliotransmitters through astroglial connexin 43 hemichannels is necessary for fear memory consolidation in the basolateral amygdala. FASEB J. 2012;26:3649-57.

24. Klaassen LJ, Sun Z, Steijaert MN, et al. Synaptic transmission from horizontal cells to cones is impaired by loss of connexin hemichannels. PLoS Biol. 2011;9:e1001107.

25. Contreras JE, Saez JC, Bukauskas FF, Bennett MV. Gating and regulation of connexin $43(\mathrm{Cx} 43)$ hemichannels. Proceedings of the National Academy of Sciences of the United States of America. 2003;100:11388-93.

26. Braet K, Aspeslagh S, Vandamme W, et al. Pharmacological sensitivity of ATP release triggered by photoliberation of inositol-1,4, 5 -trisphosphate and zero extracellular calcium in brain endothelial cells. J Cell Physiol. 2003;197:205-13.

27. De Vuyst E, Wang N, Decrock E, et al. $\mathrm{Ca}(2+)$ regulation of connexin 43 hemichannels in C6 glioma and glial cells. Cell Calcium. 2009;46:176-87.

28. Thimm J, Mechler A, Lin H, Rhee S, Lal R. Calcium-dependent open/closed conformations and interfacial energy maps of reconstituted hemichannels. The Journal of biological chemistry. 2005;280:10646-54.

29. John SA, Kondo R, Wang SY, Goldhaber JI, Weiss JN. Connexin43 hemichannels opened by metabolic inhibition. The Journal of biological chemistry. 1999;274:236-40.

30. Contreras JE, Sanchez HA, Eugenin EA, et al. Metabolic inhibition induces opening of unapposed connexin 43 gap junction hemichannels and reduces gap junctional communication in cortical astrocytes in culture. Proceedings of the National Academy of Sciences of the United States of America. 2002;99:495-500.

31. Retamal MA, Cortes CJ, Reuss L, Bennett MV, Saez JC. Snitrosylation and permeation through connexin 43 hemichannels in astrocytes: induction by oxidant stress and reversal by reducing agents. Proceedings of the National Academy of Sciences of the United States of America. 2006;103:4475-80

32. Wang N, De Bock M, Antoons G, et al. Connexin mimetic peptides inhibit $\mathrm{C} \times 43$ hemichannel opening triggered by voltage and intracellular Ca2+ elevation. Basic research in cardiology. 2012;107:304.

33. Ebihara L. New roles for connexons. News Physiol Sci. 2003;18: 100-3.

34. Quist AP, Rhee SK, Lin H, Lal R. Physiological role of gap-junctional hemichannels. Extracellular calcium-dependent isosmotic volume regulation. The Journal of cell biology. 2000;148:1063-74.

35. Froger N, Orellana JA, Calvo CF, et al. Inhibition of cytokineinduced connexin 43 hemichannel activity in astrocytes is neuroprotective. Mol Cell Neurosci. 2010;45:37-46.

36. De Bock M, Culot M, Wang N, et al. Connexin channels provide a target to manipulate brain endothelial calcium dynamics and blood-brain barrier permeability. J Cereb Blood Flow Metab. 2011;31:1942-57.

37. Danesh-Meyer HV, Kerr NM, Zhang J, et al. Connexin43 mimetic peptide reduces vascular leak and retinal ganglion cell death following retinal ischaemia. Brain : a journal of neurology. 2012;135:506-20.

38. Gomes P, Srinivas SP, Van Driessche W, Vereecke J, Himpens B. ATP release through connexin hemichannels in corneal endothelial cells. Invest Ophthalmol Vis Sci. 2005;46:1208-18.

39. Di Virgilio F, Sanz JM, Chiozzi P, Falzoni S. The P2Z/P2X7 receptor of microglial cells: a novel immunomodulatory receptor. Prog Brain Res. 1999;120:355-68.

40. Kim Y, Davidson JO, Gunn KC, et al. Role of Hemichannels in CNS Inflammation and the Inflammasome Pathway. Adv Protein Chem Struct Biol. 2016;104:1-37.
41. Cotrina ML, Lin JH, Lopez-Garcia JC, Naus CC, Nedergaard M. ATP-mediated glia signaling. The Journal of neuroscience : the official journal of the Society for Neuroscience. 2000;20:2835-44.

42. Huang C, Han X, Li X, et al. Critical role of connexin 43 in secondary expansion of traumatic spinal cord injury. The Journal of neuroscience : the official journal of the Society for Neuroscience. 2012;32:3333-8.

43. O'Carroll SJ, Gorrie CA, Velamoor S, Green CR, Nicholson LF. Connexin43 mimetic peptide is neuroprotective and improves function following spinal cord injury. Neurosci Res. 2013;75: 256-67.

44. Mao Y, Tonkin RS, Nguyen T, et al. Systemic Administration of Connexin43 Mimetic Peptide Improves Functional Recovery after Traumatic Spinal Cord Injury in Adult Rats. J Neurotrauma. 2016.

45. Clarke TC, Williams OJ, Martin PE, Evans WH. ATP release by cardiac myocytes in a simulated ischaemia model: inhibition by a connexin mimetic and enhancement by an antiarrhythmic peptide. Eur J Pharmacol. 2009;605:9-14.

46. Davidson JO, Green CR, Nicholson LF, et al. Connexin hemichannel blockade improves outcomes in a model of fetal ischemia. Annals of neurology. 2012;71:121-32.

47. Davidson JO, Drury PP, Green CR, et al. Connexin hemichannel blockade is neuroprotective after asphyxia in preterm fetal sheep. PLoS One. 2014;9:e96558.

48. Theodoric N, Bechberger JF, Naus CC, Sin WC. Role of gap junction protein connexin 43 in astrogliosis induced by brain injury. PLoS One. 2012;7:e47311.

49. Chen YS, Green CR, Danesh-Meyer HV, Rupenthal ID. Neuroprotection in the treatment of glaucoma - A focus on connexin43 gap junction channel blockers. Eur J Pharm Biopharm. 2015.

50. Guo CX, Mat Nor MN, Danesh-Meyer HV, et al. Connexin43 Mimetic Peptide Improves Retinal Function and Reduces Inflammation in a Light-Damaged Albino Rat Model. Invest Ophthalmol Vis Sci. 2016;57:3961-73.

51. Decrock E, De Vuyst E, Vinken M, et al. Connexin 43 hemichannels contribute to the propagation of apoptotic cell death in a rat C6 glioma cell model. Cell Death Differ. 2009;16:151-63.

52. Chen G, Park CK, Xie RG, et al. Connexin-43 induces chemokine release from spinal cord astrocytes to maintain late-phase neuropathic pain in mice. Brain : a journal of neurology. 2014;137: 2193-209.

53. Cronin M, Anderson PN, Cook JE, Green CR, Becker DL. Blocking connexin 43 expression reduces inflammation and improves functional recovery after spinal cord injury. Mol Cell Neurosci. 2008;39:152-60.

54. Guo CX, Tran H, Green CR, Danesh-Meyer HV, Acosta ML. Gap junction proteins in the light-damaged albino rat. Molecular vision. 2014;20:670-82.

55. Theriault E, Frankenstein UN, Hertzberg EL, Nagy JI. Connexin 43 and astrocytic gap junctions in the rat spinal cord after acute compression injury. The Journal of comparative neurology. 1997;382:199-214.

56. O'Carroll SJ, Alkadhi M, Nicholson LF, Green CR. Connexin 43 mimetic peptides reduce swelling, astrogliosis, and neuronal cell death after spinal cord injury. Cell communication \& adhesion. 2008; 15:27-42.

57. Braet K, Vandamme W, Martin PE, Evans WH, Leybaert L. Photoliberating inositol-1,4,5-trisphosphate triggers ATP release that is blocked by the connexin mimetic peptide gap 26. Cell Calcium. 2003:33:37-48.

58. Wang N, De Vuyst E, Ponsaerts R, et al. Selective inhibition of Cx43 hemichannels by Gap19 and its impact on myocardial ischemia/reperfusion injury. Basic research in cardiology. 2013;108:309. 
59. Coutinho P, Qiu C, Frank S, et al. Limiting burn extension by transient inhibition of Connexin43 expression at the site of injury. British journal of plastic surgery. 2005;58:658-67.

60. Qiu C, Coutinho P, Frank S, et al. Targeting connexin43 expression accelerates the rate of wound repair. Current biology : CB. 2003; 13:1697-703.

61. Ormonde S, Chou CY, Goold L, et al. Regulation of connexin 43 gap junction protein triggers vascular recovery and healing in human ocular persistent epithelial defect wounds. The Journal of membrane biology. 2012;245:381-8.

62. Guan Z, Buckman SY, Pentland AP, Templeton DJ, Morrison AR. Induction of cyclooxygenase-2 by the activated MEKK1 SEK1/ MKK4 p38 mitogen-activated protein kinase pathway. The Journal of biological chemistry. 1998;273:12901-8.

63. O'Carroll SJ, Kho DT, Wiltshire R, et al. Pro-inflammatory TNFalpha and IL-1beta differentially regulate the inflammatory phenotype of brain microvascular endothelial cells. Journal of neuroinflammation. 2015;12:131.

64. Chekeni FB, Elliott MR, Sandilos JK, et al. Pannexin 1 channels mediate 'find-me' signal release and membrane permeability during apoptosis. Nature. 2010;467:863-7.

65. Bondarenko A, Chesler M. Calcium dependence of rapid astrocyte death induced by transient hypoxia, acidosis, and extracellular ion shifts. Glia. 2001;34:143-9.

66. Silverman WR, de Rivero Vaccari JP, Locovei S, et al. The pannexin 1 channel activates the inflammasome in neurons and astrocytes. The Journal of biological chemistry. 2009;284:18143-51.

67. Kaneda M, Farrant M, Cull-Candy SG. Whole-cell and singlechannel currents activated by GABA and glycine in granule cells of the rat cerebellum. The Journal of physiology. 1995;485 ( Pt 2): 419-35.

68. Qin H, Shao Q, Igdoura SA, Alaoui-Jamali MA, Laird DW. Lysosomal and proteasomal degradation play distinct roles in the life cycle of $\mathrm{Cx} 43$ in gap junctional intercellular communicationdeficient and -competent breast tumor cells. The Journal of biological chemistry. 2003;278:30005-14.

69. White MP, Fisher LJ. Degree of light damage to the retina varies with time of day of bright light exposure. Physiology \& behavior. 1987;39:607-13.

70. Yu TY, Acosta ML, Ready S, Cheong YL, Kalloniatis M. Light exposure causes functional changes in the retina: increased photoreceptor cation channel permeability, photoreceptor apoptosis, and altered retinal metabolic function. Journal of neurochemistry. 2007;103:714-24.

71. Kim Y, Green CR. Assessing Connexin Hemichannel Function during Ischemic Injury and Reperfusion. In: Bai D, Saez JC, editors. Gap Junction Channels and Hemichannels. Boca Raton, FL: CRC Press; 2016. p. 169-88.

72. Kang J, Kang N, Lovatt D, et al. Connexin 43 hemichannels are permeable to ATP. The Journal of neuroscience : the official journal of the Society for Neuroscience. 2008;28:4702-11.

73. el-Fouly MH, Trosko JE, Chang CC. Scrape-loading and dye transfer. A rapid and simple technique to study gap junctional intercellular communication. Experimental cell research. 1987;168:422-30.

74. Hutnik CM, Pocrnich CE, Liu H, Laird DW, Shao Q. The protective effect of functional connexin 43 channels on a human epithelial cell line exposed to oxidative stress. Invest Ophthalmol Vis Sci. 2008;49:800-6.

75. Das Sarma J, Meyer RA, Wang F, et al. Multimeric connexin interactions prior to the trans-Golgi network. Journal of cell science. 2001;114:4013-24

76. Hansen AJ. Effect of anoxia on ion distribution in the brain. Physiological reviews. 1985;65:101-48.
77. Rosner MJ, Becker DP. Experimental brain injury: successful therapy with the weak base, tromethamine. With an overview of CNS acidosis. Journal of neurosurgery. 1984;60:961-71.

78. Bondarenko A, Chesler M. Rapid astrocyte death induced by transient hypoxia, acidosis, and extracellular ion shifts. Glia. 2001;34: $134-42$.

79. Godecke S, Roderigo C, Rose CR, et al. Thrombin-induced ATP release from human umbilical vein endothelial cells. Am J Physiol Cell Physiol. 2012;302:C915-23.

80. Weilinger NL, Tang PL, Thompson RJ. Anoxia-induced NMDA receptor activation opens pannexin channels via Src family kinases. The Journal of neuroscience : the official journal of the Society for Neuroscience. 2012;32:12579-88.

81. Hartings JA, Rolli ML, Lu XC, Tortella FC. Delayed secondary phase of peri-infarct depolarizations after focal cerebral ischemia: relation to infarct growth and neuroprotection. The Journal of neuroscience : the official journal of the Society for Neuroscience. 2003;23:11602-10.

82. Ebihara L, Steiner E. Properties of a nonjunctional current expressed from a rat connexin 46 cDNA in Xenopus oocytes. The Journal of general physiology. 1993;102:59-74.

83. Paul DL, Ebihara L, Takemoto LJ, Swenson KI, Goodenough DA. Connexin46, a novel lens gap junction protein, induces voltagegated currents in nonjunctional plasma membrane of Xenopus oocytes. The Journal of cell biology. 1991;115:1077-89.

84. Willebrords J, Crespo Yanguas S, Maes M, et al. Connexins and their channels in inflammation. Crit Rev Biochem Mol Biol. 2016:1-27.

85. Lipmann F. Metabolic generation and utilization of phosphate bond energy. Adv Enzymol Relat Areas Mol Biol. 1941;1:99-162.

86. Melani A, Amadio S, Gianfriddo M, et al. P2X7 receptor modulation on microglial cells and reduction of brain infarct caused by middle cerebral artery occlusion in rat. J Cereb Blood Flow Metab. 2006;26:974-82.

87. During MJ, Spencer DD. Adenosine: a potential mediator of seizure arrest and postictal refractoriness. Annals of neurology. 1992;32:618-24.

88. Salter MW, Hicks JL. ATP-evoked increases in intracellular calcium in neurons and glia from the dorsal spinal cord. The Journal of neuroscience : the official journal of the Society for Neuroscience. 1994;14:1563-75.

89. Eltzschig HK, Eckle T, Mager A, et al. ATP release from activated neutrophils occurs via connexin 43 and modulates adenosinedependent endothelial cell function. Circ Res. 2006;99:1100-8.

90. Yamamoto K, Korenaga R, Kamiya A, et al. P2X(4) receptors mediate ATP-induced calcium influx in human vascular endothelial cells. Am J Physiol Heart Circ Physiol. 2000;279:H285-92.

91. Kumar H, Kumagai Y, Tsuchida T, et al. Involvement of the NLRP3 inflammasome in innate and humoral adaptive immune responses to fungal beta-glucan. Journal of immunology. 2009;183:8061-7.

92. Martinon F, Agostini L, Meylan E, Tschopp J. Identification of bacterial muramyl dipeptide as activator of the NALP3/cryopyrin inflammasome. Current biology : CB. 2004;14:1929-34.

93. Agostini L, Martinon F, Burns K, et al. NALP3 forms an IL-1betaprocessing inflammasome with increased activity in MuckleWells autoinflammatory disorder. Immunity. 2004;20:319-25.

94. Tschoeke SK, Oberholzer A, Moldawer LL. Interleukin-18: a novel prognostic cytokine in bacteria-induced sepsis. Critical care medicine. 2006;34:1225-33.

95. Hennessy E, Griffin EW, Cunningham C. Astrocytes Are Primed by Chronic Neurodegeneration to Produce Exaggerated Chemokine and Cell Infiltration Responses to Acute Stimulation with the Cytokines IL-1beta and TNF-alpha. The Journal of neuroscience : the official journal of the Society for Neuroscience. 2015;35:841122. 
96. Marc RE, Jones BW, Watt CB, et al. Extreme retinal remodeling triggered by light damage: implications for age related macular degeneration. Molecular vision. 2008;14:782-806.

97. Parsons AA, Bingham S, Raval P, et al. Tonabersat (SB-220453) a novel benzopyran with anticonvulsant properties attenuates trigeminal nerve-induced neurovascular reflexes. British journal of pharmacology. 2001;132:1549-57.

98. Upton N, Blackburn TP, Campbell CA, et al. Profile of SB204269, a mechanistically novel anticonvulsant drug, in rat models of focal and generalized epileptic seizures. British journal of pharmacology. 1997;121:1679-86.

99. Lee IH, Lindqvist E, Kiehn O, Widenfalk J, Olson L. Glial and neuronal connexin expression patterns in the rat spinal cord during development and following injury. The Journal of comparative neurology. 2005;489:1-10.

100. Ohsumi A, Nawashiro H, Otani N, et al. Temporal and spatial profile of phosphorylated connexin 43 after traumatic brain injury in rats. J Neurotrauma. 2010;27:1255-63.

101. Haupt C, Witte OW, Frahm C. Up-regulation of Connexin 43 in the glial scar following photothrombotic ischemic injury. Mol Cell Neurosci. 2007;35:89-99.

102. Fonseca CG, Green CR, Nicholson LF. Upregulation in astrocytic connexin 43 gap junction levels may exacerbate generalized seizures in mesial temporal lobe epilepsy. Brain research. 2002;929: 105-16.

103. Vis JC, Nicholson LF, Faull RL, et al. Connexin expression in Huntington's diseased human brain. Cell biology international. 1998;22:837-47.

104. De Vuyst E, Decrock E, Cabooter L, et al. Intracellular calcium changes trigger connexin 32 hemichannel opening. EMBO J. 2006;25:34-44.

105. De Vuyst E, Decrock E, De Bock M, et al. Connexin hemichannels and gap junction channels are differentially influenced by lipopolysaccharide and basic fibroblast growth factor. Mol Biol Cell. 2007;18:34-46.

106. Kielian T. Glial connexins and gap junctions in CNS inflammation and disease. Journal of neurochemistry. 2008;106:1000-16.
107. Retamal MA, Froger N, Palacios-Prado N, et al. Cx43 hemichannels and gap junction channels in astrocytes are regulated oppositely by proinflammatory cytokines released from activated microglia. The Journal of neuroscience : the official journal of the Society for Neuroscience. 2007;27:13781-92.

108. Torres A, Wang F, Xu Q, et al. Extracellular $\mathrm{Ca}(2)(+)$ acts as a mediator of communication from neurons to glia. Sci Signal. 2012; 5:ra8.

109. Cea LA, Balboa E, Puebla C, et al. Dexamethasone-induced muscular atrophy is mediated by functional expression of connexinbased hemichannels. Biochim Biophys Acta. 2016;1862:1891-9.

110. Takeuchi H, Mizoguchi H, Doi Y, et al. Blockade of gap junction hemichannel suppresses disease progression in mouse models of amyotrophic lateral sclerosis and Alzheimer's disease. PLoS One. 2011;6:e21108.

111. Kozoriz MG, Bechberger JF, Bechberger GR, et al. The connexin43 C-terminal region mediates neuroprotection during stroke. J Neuropathol Exp Neurol. 2010;69:196-206.

112. Kozoriz MG, Lai S, Vega JL, et al. Cerebral ischemic injury is enhanced in a model of oculodentodigital dysplasia. Neuropharmacology. 2013;75:549-56.

113. Chen Y, Green CR, Wang K, Danesh-Meyer HV, Rupenthal ID. Sustained intravitreal delivery of connexin 43 mimetic peptide by poly(d,l-lactide-co-glycolide) acid micro- and nanoparticles Closing the gap in retinal ischaemia. Eur J Pharm Biopharm. 2014.

114. Yi C, Mei X, Ezan P, et al. Astroglial connexin 43 contributes to neuronal suffering in a mouse model of Alzheimer's disease. Cell Death Differ. 2016;23:1691-701.

115. Choi SR, Roh DH, Yoon SY, et al. Astrocyte sigma-1 receptors modulate connexin 43 expression leading to the induction of below-level mechanical allodynia in spinal cord injured mice. Neuropharmacology. 2016;111:34-46.

116. Zhang J, O'Carroll S, Danesh-Meyer H, et al. Connexin-based therapeutic approaches to inflammation in the central nervous system. In: Oviedo-Orta E, Kwak B, Evans W, editors. Connexin Cell Communication Channels: Roles in the immune system and immunopathology. Boca Raton, Florida: Taylor and Francis Group, CRC Press. ; 2013. p. 273-305. 